\title{
Bulk gauge and matter fields in nested warping: II. Symmetry breaking and phenomenological consequences
}

\author{
Mathew Thomas Arun ${ }^{1}$ and Debajyoti Choudhury \\ Department of Physics and Astrophysics, University of Delhi, \\ Delhi 11000\%, India \\ E-mail: thomas.mathewarun@gmail.com, debajyoti.choudhury@gmail.com
}

ABSTRACT: Generalizing the Randall-Sundrum scenario to higher dimensions with nested warpings has been shown to avoid the constraints besetting the former. In the first paper of this series [JHEP 09 (2015) 202], the Standard Model gauge and fermion fields were extended into such a six-dimensional bulk and the construction was shown to have several interesting and welcome features. In this paper, we discuss the electroweak symmetry breaking, presenting a novel Higgs localization mechanism that leads to interesting phenomenology in the Higgs sector. Localizing the Higgs modifies the $Z_{\mu}$ and $W_{\mu}$ boson wavefunctions, which leads to tree level changes in the oblique parameters. Using these as well as the correction to low-energy four-Fermi operators, we derive the constraints on our model and also discuss the gauge coupling evolution therein. Amusingly, the model can naturally incorporate a Higgs resonance in the $700-800 \mathrm{GeV}$ range.

KEYwords: Phenomenology of Field Theories in Higher Dimensions

ArXiv EPrint: 1601.02321

\footnotetext{
${ }^{1}$ Corresponding author.
} 


\section{Contents}

1 Introduction 1

2 Gauge and fermion fields 3

$\begin{array}{llr}3 & \text { Higgs } & \mathbf{8}\end{array}$

3.1 The Higgs spectrum $\quad 9$

3.2 Corrections to the Higgs potential and modifications to the spectrum 11

$\begin{array}{lll}3.3 & \text { An alternative scheme } & 12\end{array}$

4 Effective Lagrangian $\quad 14$

$\begin{array}{ll}\text { 4.1 The oblique parameters } & 16\end{array}$

$\begin{array}{lll}4.2 G_{f} & 17\end{array}$

5 Confronting electroweak precision measurements $\quad 18$

$\begin{array}{llr}6 & \text { Beta function } & 19\end{array}$

$\begin{array}{lll}7 & \text { Summary and outlook } & 22\end{array}$

\section{Introduction}

It has long been recognized that theories defined in dimensions larger than four may provide geometric resolutions to some of the quandaries faced by the Standard Model. Amidst diverse theoretical constructs addressing such issues, have been efforts [1-9] to intertwine gravity with low energy phenomenology. The Randall-Sundrum (RS) model [6] and its extensions comprise one such set of endeavours. Unlike in the ADD scenario [8], wherein the hierarchy is sought to be explained by introducing a large volume in the extra dimensions, in the RS model it is done by postulating a nonfactorizable geometry with an exponential warping between two flat 3-branes. While we are located on the $\mathrm{TeV}$ brane (wherein the natural scale of the theory, viz. $M_{\mathrm{Pl}}$, is warped down to and perceived as the $\mathrm{TeV}$ scale), the other (Planck) brane remains hidden.

A more interesting (from the particle physics point of view) model is constructed by a minimal extension of this RS model with gauge bosons in the bulk and fermions stuck to the brane $[10,11]$. Such forays into the bulk come at a cost, though. For example, the gauge boson KK-excitations couple to fermion bilinears almost universally and with a strength approximately eight times as large as that of the zero mode. This result, in conjunction with the global fits on the four Fermi operator [12], demand that the first excited mode mass must be $>23 \mathrm{TeV}$. To appreciate this constraint, it is useful to reexpress it in terms of the model parameters, viz. the fundamental five-dimensional mass $M_{5 D}$, the radius of 
compactification $R_{y}$ and the exponential $\left(e^{-c|y|}\right)$ warping parameter $c$, whereby it translates to $c /\left(R_{y} M_{5 D}\right)>4.5$. On the other hand, the very applicability of semi-classical arguments, on which the entire RS construction hinges, calls for this combination to be $\lesssim 0.1$, thus calling into question the trustworthiness of this approach.

On allowing the fermions too to enter the bulk, it was shown [13] that the coupling of the fermion zero-mode to the first KK gauge boson could be suppressed significantly, thereby relaxing the constraints from the four-Fermi operator. On the other hand, since the mass hierarchy problem can be solved only by using a TeV-brane localized Higgs field, the latter's gauge coupling deforms the boundary conditions on the gauge bosons. The consequent distortion in the profile of the lowest gauge boson, results in tree level corrections to the electroweak oblique parameters [14]. Consistency with the precision data now demands that the first KK-mode for the gauge boson be heavier than $27 \mathrm{TeV}$ (or, equivalently, $c R_{y}^{-1} e^{-c \pi}>11 \mathrm{TeV}$ ), thereby resurrecting the problem in a different guise. This, though, could be cured, albeit at the expense of introducing a custodial symmetry in the bulk [15]. The enhanced gauge structure preserves the isospin symmetry and thus softens the constraint on the $T$-parameter. Similarly, localizing the light fermions near the Planck-brane controls the $S$-parameter, such that the precision test data fits are satisfied by a KK gauge boson with mass of a few TeVs.

On a track parallel to this, emerged several attempts in creating models in $(5+1)$ dimensions. While the flat space variants [16-24] did consider bulk matter fields so as to address some of the lacunae of the SM, those with non-factorizable geometries [25, 28-30, 34, 38, 41, 45-48] typically restricted themselves to discussion of the hierarchy and/or cosmological issues. Although seemingly modest in their aspiration, the latter set of constructions have recently gained relevance in the context of negative results achieved by both the ATLAS [49] for RS graviton resonances. While reasonable values for the ratio of the five-dimensional curvature and the fundamental mass scale would predict that the mass of the first KK-graviton be a few times larger than that of the Higgs, the current lower limit of $\sim 2.66 \mathrm{TeV}$ (at 95\% C.L.) is already causing some tension for the scenario. In ref. [51], though, it was demonstrated that, in the event of nested warping in a $6 \mathrm{D}$ scenario, the graviton modes comfortably evade the current bounds from the LHC. Furthermore, as ref. [52] points out, not only is the allowed parameter space of the model quite extensive and can be probed well in the current run of LHC, it also admits an explanation of the recently reported anomaly $[53,54]$ at $m_{\gamma \gamma} \sim 750 \mathrm{GeV}$.

It is, thus, interesting to consider the possibility of allowing the SM fields into the bulk of such a nested warping scenario, and we had developed this formalism in ref. [55], hereafter referred to as Paper I. The construction has several striking features. The most notable is that, apart from offering an "explanation" of the number of fermion generations, it essentially "localizes" part of the fermions onto a 4-brane. This has the immediate consequence that whereas the gauge bosons (and, of course, the graviton) have a "tower of KK-towers", for the fermions one of the towers is missing. This would have striking ramifications in collider searches, both in terms of the observed low-energy spectrum as well as in the decay patterns (and, hence, in the signature topologies). Furthermore, the "missing" fermionic states would leave imprint in both corrections to observables as 
well as in engendering rare processes. In the present work, we examine some of such phenomenological consequences.

The rest of this paper is structured as follows. We start out with a brief recapitulation of the scenario augmented by a discussion of fermion mixing (an aspect that was glossed over earlier). Subsequently, in section 3, we consider the Higgs sector in detail and present the Higgs spectrum for the particular localization that we employ. This is followed, in section 4 , by the derivation of an effective Lagrangian that allows us to reliably calculate four-Fermi operators as well as the electroweak precision observables. Utilizing this, in section 5, to constrain the parameter space, we next investigate (in section 6) the renormalization group flow of the gauge couplings, which allows us examine the nature of gauge unification. Finally, we summarise in section 7 .

\section{Gauge and fermion fields}

We consider a six-dimensional space-time compactified down to four dimensions with a $Z_{2}$ orbifolding in each of the two extra dimensions, viz. $M^{1,5} \rightarrow\left[M^{1,3} \times S^{1} / Z_{2}\right] \times S^{1} / Z_{2}$. A successive (nested) warping is assumed leaving the four-dimensional space to be flat. In other words, the line element is of the form [48]

$$
d s^{2}=b^{2}\left(x_{5}\right)\left[a^{2}\left(x_{4}\right) \eta_{\mu \nu} d x^{\mu} d x^{\nu}+R_{y}^{2} d x_{4}^{2}\right]+r_{z}^{2} d x_{5}^{2},
$$

where the compact directions are represented by the dimensionless coordinates $x_{4,5} \in[0, \pi]$ with $R_{y}$ and $r_{z}$ being the corresponding moduli. The background geometry is given by the six-dimensional Einstein-Hilbert action (with a natural scale $M_{6}$ ) and a negative (sixdimensional) cosmological constant $\Lambda_{6}$ yielding [48]

$$
\begin{array}{ll}
a\left(x_{4}\right)=e^{-c\left|x_{4}\right|} & c=\frac{R_{y} k}{r_{z} \cosh k \pi} \equiv \frac{\aleph k}{\cosh (k \pi)} \\
b\left(x_{5}\right)=\frac{\cosh \left(k x_{5}\right)}{\cosh (k \pi)} & k=r_{z} \sqrt{\frac{-\Lambda_{6}}{10 M_{6}^{4}}} \equiv \epsilon r_{z} M_{6} .
\end{array}
$$

The difference in scale between the Planck brane and the TeV brane, where the Higgs is localized, sets a measure for $w$, the extent of the hierarchy. Typically, $w$ ranges from $e^{-c \pi}$ to $e^{-c \pi}$ sech $k \pi$, with the exact value depending on the details of the Higgs localization.

Clearly, we can consistently neglect quantum corrections to the bulk gravity action (necessary for the validity of the semi classical treatment) only if the bulk curvature is significantly smaller than the fundamental scale $M_{6}$, or in other words if $\epsilon \lesssim 0.1$. On the other hand, the requirement of not reintroducing a large hierarchy requires that $\aleph$ (the ratio of the two moduli) should not be too large. This, along with the phenomenological requirement of $w \sim 10^{-16}$ (or even an order of magnitude or two larger) forces the theory into one of two branches, namely $(i) c \sim \mathcal{O}(10), k \lesssim 1$ or $(i i) k \sim \mathcal{O}(10)$ and a negligibly small $c$ [48]. While each branch has its merits, the second one results in considerably enhanced couplings for the KK-gravitons [51]. Furthermore, once gauge fields are allowed to go into the bulk, their KK-excitations, for this branch of the theory, are bestowed with 
too large a coupling to admit perturbation theory [55]. Consequently, we shall concentrate on the first branch alone.

We start our review of the SM fields with the gauge sector, which, along with the fermions, percolates fully into the bulk. The kinetic term, for a theory with unbroken symmetry, is thus given by

$$
\begin{aligned}
\mathcal{L} & =\frac{-1}{4} \sqrt{-g} F_{M N} F^{M N}+\mathcal{L}_{g f} \\
\mathcal{L}_{g f} & =\frac{-\sqrt{-g}}{2 \zeta}\left[g^{\mu \nu}\left\{\partial_{\mu} A_{\nu}-\frac{\zeta}{2}\left(\Gamma_{\mu \nu}^{4} A_{4}+\Gamma_{\mu \nu}^{5} A_{5}\right)\right\}+\zeta\left(g^{44} D_{4} A_{4}+g^{55} D_{5} A_{5}\right)\right]^{2} \\
& =\frac{-R_{y} r_{z} b}{2 \zeta}\left[\eta^{\mu \nu} \partial_{\mu} A_{\nu}+\frac{\zeta}{b}\left(\partial_{4} \frac{a^{2} b A_{4}}{R_{y}^{2}}+\partial_{5} \frac{a^{2} b^{3} A_{5}}{r_{z}^{2}}\right)\right]^{2},
\end{aligned}
$$

where the choice of the gauge-fixing term (a curved-space analog of the generalized $R_{\zeta}$ gauge) eliminates the cumbersome kinetic mixing terms between $A_{\mu}$ and $A_{4,5}$. Writing $A_{\mu}$ in terms of the KK modes, viz.

$$
A_{\kappa}=\frac{1}{\sqrt{R_{y} r_{z}}} \sum_{n, p} A_{\kappa}^{(n, p)}\left(x^{\mu}\right) \eta_{n, p}\left(x_{4}\right) \chi_{p}\left(x_{5}\right)
$$

with $\eta_{n, p}$ and $\chi_{p}$ normalized as

$$
\int b\left(x_{5}\right) \chi_{p_{1}} \chi_{p_{2}} d x_{5}=\delta_{p_{1}, p_{2}}, \quad \int \eta_{n_{1}, p} \eta_{n_{2}, p} d x_{4}=\delta_{n_{1}, n_{2}},
$$

the solutions for the modes are

$$
\begin{aligned}
\chi_{p}\left(x_{5}\right) & =\frac{1}{B} \operatorname{sech}^{3 / 2}\left(k x_{5}\right)\left(c_{1} P_{\nu_{p}}^{3 / 2}\left(\tanh k x_{5}\right)+c_{2} Q_{\nu_{p}}^{3 / 2}\left(\tanh k x_{5}\right)\right) \\
\eta_{n, p}\left(x_{4}\right) & =\frac{e^{c\left|x_{4}\right|}}{N}\left(J_{\nu_{n}}\left(y_{n}\right)+c_{n p} Y_{\nu_{n}}\left(y_{n}\right)\right) \\
y_{n} & \equiv m_{n p} \frac{r_{z}}{k} e^{c\left|x_{4}\right|} \cosh (k \pi)=m_{n p} \frac{R_{y}}{c} e^{c\left|x_{4}\right|} \\
\nu_{n} & =\sqrt{1+\frac{r_{z}^{2}}{k} m_{p}^{2} \cosh ^{2}(k \pi)} \\
\nu_{p} & =\frac{-1}{2}+\nu_{n} .
\end{aligned}
$$

Before we impose the boundary conditions on the $\chi$ 's and the $\eta$ 's (and, thereby, compute the spectrum), let us remind ourselves that the electroweak symmetry has, of course, to be broken spontaneously. While this could, in principle, be done with a bulk Higgs field, such a course of action would imply that the Higgs mass (or the vacuum expectation value) would assume the natural scale, namely $M_{6}$, and the hierarchy problem would resurface. This is exactly analogous to the case of the corresponding five-dimensional scenario. A way out would be to confine the Higgs to a brane wherein the perceived scale is naturally low. In the present case, it could be 3 -brane located at $\left(x_{4}=\pi, x_{5}=0\right)$, or, more generally, the 
4-brane at $x_{4}=\pi$. As has been pointed out in ref. [55], the first course of action leads to a equation of motion for the gauge bosons that does not let itself to a closed-form solution commensurate with the boundary conditions. To this end, we consider a theory with an explicit cutoff $\lesssim R_{y}^{-1}$ and described by a Higgs Lagrangian of the form ${ }^{1}$

$$
\begin{aligned}
& \mathcal{L}_{h}=\delta\left(x_{4}-\pi\right) \sqrt{-g_{5}}\left(g^{\mu \nu} D_{\mu} \phi\left(x^{\bar{M}}\right)^{\dagger} D_{\nu} \phi\left(x^{\bar{M}}\right)+\aleph^{-2} g^{55}\left|D_{5} \phi\left(x^{\bar{M}}\right)\right|^{2}+V(\phi)\right), \\
& D_{\mu}=\partial_{\mu}-i g_{\mathrm{YM}} A_{\mu}\left(x_{\nu}, x_{4}, x_{5}\right)
\end{aligned}
$$

where the barred indices ( $\bar{M}$ etc.) run over the coordinates $(0,1,2,3,5)$ relevant to this brane. Note that the form of the Lagrangian is slightly different from that proposed in ref. [55]. In particular, the factor $\aleph^{-2}$ ensures that the natural scale of the theory is $R_{y}^{-1}$ and not $r_{z}^{-1}$ (which is larger than the cutoff). While the form above is seemingly inconsistent with the full five-dimensional Lorentz invariance, this is not of concern here. In fact, the very presence of the $x_{5}$-dependent brane tension $V_{2}\left(x_{5}\right)[48,55]$ has already destroyed part of the symmetry leaving behind a manifest four-dimensional Lorentz invariance. $V(\phi)$ is a potential admitting a nontrivial vacuum and, thus, a brane-localized mass term for the gauge boson. The solution to the corresponding gauge equation of motion is still rather complicated, but can be simplified substantially if $V(\phi)$ is such that the scalar equation of motion admits a $x_{5}$-dependent profile of the form $\left\langle\phi\left(x_{5}\right)\right\rangle \propto v / \sqrt{b\left(x_{5}\right)}$ with $v$ being the (constant) vacuum expectation value as mentioned in ref. [55]. Postponing discussions about the form of the $V(\phi)$ needed, we assume that the profile is indeed so. This would, then, introduce a brane-localized gauge field mass term of the form

$$
\mathcal{L}_{m}=\frac{\sqrt{-g_{5}}}{2} \widetilde{M}^{2}\left(x_{5}\right) g_{5}^{\mu \nu} A_{\mu} A_{\nu} \delta\left(x_{4}-\pi\right)
$$

with $\widetilde{M}=m / \sqrt{b\left(x_{5}\right)}$ where $m \propto g_{\mathrm{YM}} v$. The consequent boundary conditions are

$$
\left.\chi_{p}^{\prime}\right|_{x_{5}=0}=0=\left.\chi_{p}^{\prime}\right|_{x_{5}=\pi}
$$

and

$$
\left.\eta_{n, p}^{\prime}\right|_{x_{4}=0}=0, \quad \text { and }\left.\quad \eta_{n, p}^{\prime}\right|_{x_{4}=\pi}=m^{2} R_{y}^{2} \eta_{n, p}(\pi) .
$$

For $m_{p=0}=0$, we have, for the modes $\eta_{n 0}$,

$$
J_{0}\left(e^{-c \pi} \alpha_{n 0}\right)\left(2 c \alpha_{n 0} Y_{0}\left(\alpha_{n 0}\right)+R_{y}^{2} m^{2} Y_{1}\left(\alpha_{n 0}\right)\right)=Y_{0}\left(e^{-c \pi} \alpha_{n 0}\right)\left(2 c \alpha_{n 0} J_{0}\left(\alpha_{n 0}\right)+R_{y}^{2} m^{2} J_{1}\left(\alpha_{n 0}\right)\right),
$$

where, as before, $\alpha_{n 0} \equiv m_{n 0} R_{y} e^{c \pi} / c$. Since the lightest mass mode is to be identified with the $W / Z$ bosons, we have $\alpha_{00} \sim m_{00} R_{y} e^{c \pi} / c \ll 1$ (as $c \sim 10$ ). Expanding the Bessel functions, we obtain

$$
m_{00}^{2} \approx \frac{1}{2 \pi} m^{2} e^{-2 c \pi}
$$

Clearly, for the $W$ boson, $m^{2}=2 \pi g^{2} v^{2}$, whereas for the $Z$ boson, $m^{2}=2 \pi\left(g^{2}+g^{\prime 2}\right) v^{2}$, with $g$ and $g^{\prime}$ being the weak and hyper-charge coupling constants respectively.

\footnotetext{
${ }^{1}$ It is at this scale that the compactified direction $x_{4}$ would reveal itself and a four-dimensional description would no longer be tenable.
} 
As for the fermions, six dimensions (unlike five) admit Weyl fermions, and we just promote the SM fermions to their higher-dimensional selves. Concentrating on the positive chirality spinor $\Psi_{+}$, the Dirac Lagrangian, in terms of the sechsbeins $E_{a}^{M}$ and spin connection $w_{M}^{b c}$, is given by

$$
\mathcal{L}_{\text {Dirac }}=i \bar{\Psi}_{+} \Gamma^{a} E_{a}^{M}\left(\partial_{M}+w_{M}^{b c}\left[\Gamma_{b}, \Gamma_{c}\right]\right) \Psi_{+} .
$$

Using a representation for the gamma matrices $\Gamma_{b}$ as in ref. [55], the wavefunction $\Psi_{+}$can be expressed as

$$
\Psi_{+}=\frac{1}{\sqrt{R_{y} r_{z}}} \sum_{n, p}\left[\mathcal{F}_{+l}^{n, p}\left(x_{4}, x_{5}\right) \psi_{l}^{n, p}\left(x_{\mu}\right) \otimes \mathcal{S}_{\mathrm{up}}+\mathcal{F}_{+r}^{n, p}\left(x_{4}, x_{5}\right) \psi_{r}^{n, p}\left(x_{\mu}\right) \otimes \mathcal{S}_{\mathrm{dn}}\right]
$$

with

$$
\mathcal{S}_{\text {up }} \equiv\left(\begin{array}{ll}
1 & 0
\end{array}\right)^{T}, \quad \mathcal{S}_{\text {dn }} \equiv\left(\begin{array}{ll}
0 & 1
\end{array}\right)^{T} .
$$

A similar expression arises for $\Psi_{-}$as well. The subscripts $(l, r)$ refer to the (four-dimensional) chirality of the four-dimensional fields $\psi_{l, r}^{n, p}$. Effecting a separation of variables, the wavefunctions $\mathcal{F}_{+l / r}^{n, p}\left(x_{4}, x_{5}\right)$ can be written as

$$
\mathcal{F}_{l / r}^{n, p}\left(x_{4}, x_{5}\right)=\left[a\left(x_{4}\right)\right]^{-2}\left[b\left(x_{5}\right)\right]^{-5 / 2} \widetilde{f}_{l / r}^{n, p}\left(x_{4}\right) f_{l / r}^{p}\left(x_{5}\right)
$$

where

$$
\begin{aligned}
\widetilde{f}_{l}^{n, p}\left(x_{4}\right) & =e^{c\left|x_{4}\right| / 2}\left[c_{1} J_{\nu_{p}}\left(x_{n p} e^{c\left(\left|x_{4}\right|-\pi\right)}\right)+c_{2} Y_{\nu_{p}}\left(x_{n p} e^{c\left(\left|x_{4}\right|-\pi\right)}\right)\right] \\
\widetilde{f}_{r}^{n, p}\left(x_{4}\right) & =e^{c\left|x_{4}\right| / 2}\left[c_{3} J_{\nu_{p}}\left(x_{n p} e^{c\left(\left|x_{4}\right|-\pi\right)}\right)+c_{4} Y_{\nu_{p}}\left(x_{n p} e^{c\left(\left|x_{4}\right|-\pi\right)}\right)\right] \\
\nu_{p} & \equiv \sqrt{\frac{1}{4}+\frac{m_{p}^{2} R_{y}^{2}}{c^{2}}}=\frac{p \pi}{2 \Theta_{k}(\pi)} \\
x_{n p} & \equiv M_{n p} \frac{R_{y}}{c} e^{c \pi}
\end{aligned}
$$

and

$$
\begin{aligned}
f_{l}\left(x_{5}\right) & =\exp \left[i \kappa_{l}^{+} \Theta_{k}\left(x_{5}\right)\right]-\frac{d_{l}^{+}}{d_{l}^{-}} \exp \left[i \kappa_{l}^{-} \Theta_{k}\left(x_{5}\right)\right] \\
f_{r}\left(x_{5}\right) & =\exp \left[i \kappa_{r}^{+} \Theta_{k}\left(x_{5}\right)\right]-\exp \left[i \kappa_{r}^{-} \Theta_{k}\left(x_{5}\right)\right] \\
\Theta_{k}\left(x_{5}\right) & \equiv \tan ^{-1}\left(\tanh \frac{k x_{5}}{2}\right) .
\end{aligned}
$$

The constants $\kappa_{l / r}$ are solutions of quadratic equations, and are given by

$$
\begin{gathered}
\kappa_{r}^{ \pm}=-1 \pm \sqrt{1+4 \frac{m_{p}^{2} R_{y}^{2}}{c^{2}}} \\
\kappa_{l}^{ \pm}=1 \pm \sqrt{1+4 \frac{m_{p}^{2} R_{y}^{2}}{c^{2}}} .
\end{gathered}
$$


For the massless mode, $f_{l}^{p}\left(x_{5}\right)=1$ and $\widetilde{f}_{l}^{n, p}\left(x_{4}\right)=1$. The boundary conditions dictate that $\mathcal{F}_{+l}^{\left(n_{1}, p_{1}\right)}\left(x_{4}, x_{5}\right)=\mathcal{F}_{-r}^{\left(n_{1}, p_{1}\right)}\left(x_{4}, x_{5}\right)$ and $\mathcal{F}_{+r}^{\left(n_{1}, p_{1}\right)}\left(x_{4}, x_{5}\right)=\mathcal{F}_{-l}^{\left(n_{1}, p_{1}\right)}\left(x_{4}, x_{5}\right)$.

The Yukawa Lagrangian now sees only the brane-localized Higgs field, and can be written as

$$
\mathcal{L}_{y}=\sum_{i, j} Y_{i j} \int d^{4} x \int d x_{4} \int d x_{5} \sqrt{-g_{5}} \phi^{\dagger} D_{+}^{i}\left(x_{M}\right) S_{-}^{j}\left(x_{M}\right) \delta\left(x_{4}-\pi\right)+\text { H.c. },
$$

where $D_{+}^{i}\left(x_{M}\right)\left(S_{-}^{j}\left(x_{M}\right)\right)$ are the six-dimensional fields with chirality \pm and transforming as doublets (singlets) under $\mathrm{SU}(2)$.

In terms of the KK components, this can be re-expressed as

$$
\begin{aligned}
\mathcal{L}_{y}= & v \sum_{i, j} Y_{(+l,-r) i j}^{n_{1}, p_{1}, n_{2}, p_{2}} \int d^{4} x D_{+l}^{\left(n_{1}, p_{1}\right), i}\left(x_{\mu}\right) S_{-r}^{\left(n_{2}, p_{2}\right), j}\left(x_{\mu}\right) \\
& +v \sum_{i, j} Y_{(+r,-l) i j}^{n_{1}, p_{1}, n_{2}, p_{2}} \int d^{4} x D_{+r}^{\left(n_{1}, p_{1}\right), i}\left(x_{\mu}\right) S_{-l}^{\left(n_{2}, p_{2}\right), j}\left(x_{\mu}\right)+\text { H.c. }
\end{aligned}
$$

where the effective four-dimensional Yukawa couplings are given by

$$
\begin{aligned}
& Y_{(+l,-r) i j}^{n_{1}, p_{1}, n_{2}, p_{2}}=Y_{i j} a^{4}(\pi) \int d x_{5}\left[b\left(x_{5}\right)\right]^{9 / 2} \mathcal{F}_{+l}^{\left(n_{1}, p_{1}\right)}\left(\pi, x_{5}\right) \mathcal{F}_{-r}^{\left(n_{2}, p_{2}\right)}\left(\pi, x_{5}\right) \\
& Y_{(+r,-l) i j}^{n_{1}, p_{1}, n_{2}, p_{2}}=Y_{i, j} a^{4}(\pi) \int d x_{5}\left[b\left(x_{5}\right)\right]^{9 / 2} \mathcal{F}_{+r}^{\left(n_{1}, p_{1}\right)}\left(\pi, x_{5}\right) \mathcal{F}_{-l}^{\left(n_{2}, p_{2}\right)}\left(\pi, x_{5}\right) .
\end{aligned}
$$

Note that fermion mixing is, now, not restricted to just the usual flavour (Cabibbo) mixing, but is generalized to incorporate mixing between different KK excitations as well, both flavour-diagonal and non-diagonal. This is but a consequence of the brane-localization of the Higgs field, which breaks KK number conservation. Concentrating on the inter-level mixing, while keeping the CKM mixing in abeyance for now, clearly the former is important primarily for the heaviest flavour, viz. the top-quark. The boundary conditions ensures that the zero mode is chiral while leaving the higher modes to be vector like. The mass matrix, in the weak/KK eigenbasis $Q_{l}=\left(D_{+l}^{0,0}, D_{+l}^{1,1}, S_{-l}^{1,1}\right)$ and $Q_{r}=\left(S_{-r}^{0,0}, D_{+r}^{1,1}, S_{-r}^{1,1}\right)$, reads

$$
\mathcal{M}_{\text {top }}=\left(\begin{array}{ccc}
Y^{0,0,0,0} v & 0 & Y_{(+l,-r)}^{0,0,1,1} v \\
Y_{(+l,-r)}^{1,1,0,0} v & M_{D(1,1)} & Y_{(+l,-r)}^{1,1,1,1} v \\
0 & Y_{(-l,+r)}^{1,1,1,1} v & M_{S(1,1)}
\end{array}\right)
$$

where $M_{D(1,1)}$ and $M_{S(1,1)}$ are the tree level KK masses (in the absence of level-mixing) for the corresponding doublet and singlet fields. We have, obviously, truncated the mass matrix to the lightest nontrivial sector, so as to illustrate the salient points without unduly increasing the complexity. The physical masses are, of course, given by the eigenvalues of $\mathcal{M}_{\text {top }}^{\dagger} \mathcal{M}_{\text {top }}$. Since the doublet and singlet masses are related by a chiral rotation, $M_{D(1,1)}=-M_{S(1,1)}=M_{(1,1)}$, as calculated in Paper I. On the other hand, the very structure of the $\mathcal{F}$ 's ensure that, for a given fermion, the inter-level Yukawa couplings are, 
generically, much smaller than the same-level ones. ${ }^{2}$ In other words, $Y^{0,0,0,0} \approx Y_{(+l,-r)}^{1,1,1,1} \approx$ $Y_{(-l,+r)}^{1,1,1,1} \gg Y_{(+l,-r)}^{0,0,1,1}=Y_{(+l,-r)}^{1,1,0,0}$, with the last equality being an exact one. This makes the diagonalization of the matrix easier and, to the first order, similar to the Universal Extra Dimension scenarios, with the caveat that, in warped space, the Yukawa coupling constants are not all the same. Though the coupling increases for higher $p$ states, this is overshadowed by the increase in the tree level KK mass. And hence we could truncate the mass spectrum to $n=1, p=1$ level. On diagonalizing the above matrix numerically we get $M_{D(1,1)}^{d}=-M_{S(1,1)}^{d} \approx \sqrt{M_{1,1}^{2}+\left(\frac{Y_{(+l,-r)}^{1,1,1,1}+Y_{(-l,+r)}^{1,1,1,1}}{2} v\right)^{2}}$. For the rest of the fermions, we could safely assume $M_{D(n, p)}^{d}=-M_{S(n, p)}^{d} \approx M_{n, p}$.

\section{Higgs}

A generic 3-brane localized Higgs profile leads to equations of motion for the gauge bosons that do not admit simple closed form solutions, and this is what prompted the particular choice $^{3}$ of $\phi_{\mathrm{cl}}=v / \sqrt{r_{z} b\left(x_{5}\right)}$, in the previous section. This seemingly ad hoc ansatz is actually a $x_{5}$-dependent solution [55] of the equation of motion for a potential of the form

$$
V(\phi)=\frac{k^{2}}{R_{y}^{2}}\left[\frac{5 \operatorname{sech}^{2} k \pi}{24\left(v / \sqrt{r_{z}}\right)^{4}} \phi^{6}-\frac{7}{8} \phi^{2}\right] .
$$

Note that this potential (proportional to that in ref. [55]) is truly of the aforementioned cutoff scale.

Perturbing the scalar field about its classical value, viz. $\phi\left(x_{\mu}, x_{5}\right)=\phi_{\mathrm{cl}}\left(x_{5}\right)+\hat{\phi}\left(x_{\mu}, x_{5}\right)$, we have, for the equation of motion

$$
\frac{1}{R_{y}^{2}} \partial_{5}\left(b^{4} \partial_{5} \hat{\phi}\right)+\frac{b^{2}}{a_{\pi}^{2}} \partial_{\mu} \partial^{\mu} \hat{\phi}=\frac{k^{2}}{R_{y}^{2}}\left(\frac{25 \operatorname{sech}^{2} k \pi}{4} b^{2}-\frac{7}{4} b^{4}\right) \hat{\phi}
$$

Re-parameterizing

$$
\hat{\phi}=\frac{1}{\sqrt{r_{z}}} h_{p}\left(x_{\mu}\right) \chi_{p}^{(h)}\left(x_{5}\right)
$$

we have

$$
\begin{aligned}
\partial_{\mu} \partial^{\mu} h_{p} & =m_{p}^{2} h_{p} \\
\partial_{5}\left[b^{4} \partial_{5} \chi_{p}^{(p)}\right] & =-k^{2}\left[\gamma_{p} b^{2}+\frac{7}{4} b^{4}\right] \chi_{p}^{(h)},
\end{aligned}
$$

where

$$
m_{p}^{2}=\left(\frac{25}{4} \operatorname{sech}^{2} k \pi+\gamma_{p}\right) \frac{k^{2} a_{\pi}^{2}}{R_{y}^{2}} .
$$

Note that the nominal vacuum expectation value $v$ does not enter the expression for the masses, but the cutoff $R_{y}^{-1}$ squarely does so; and that the masses (as also $v$ ) are of the

\footnotetext{
${ }^{2}$ Note that the inter-level couplings would have vanished if the Higgs field could percolate freely into the bulk and are but a consequence of the loss of KK-number conservation brought about by the brane localization.

${ }^{3}$ Note that the factor of $r_{z}^{-1 / 2}$ is only a overall normalization and is not reflective of the natural scale of the five-dimensional theory, which would be seen to be $R_{y}^{-1}$.
} 
order of the cutoff. ${ }^{4}$ A couple of subtleties need to be considered, though. For one, the last term in eq. (3.3), namely $\frac{7}{4} k^{2} b^{4}$, could be considered a negative "bulk mass" term. Furthermore, note that $\phi_{\mathrm{cl}}$ lives entirely on one side of the nominal vev $v$. Thus, despite the positive contributions to the energy engendered by the nontrivial $x_{5}$-dependence, there is a danger of the theory admitting tachyonic modes (at least for some range of $k$ ), thereby invalidating the formulation. We shall shortly return to this.

In the regime where all $m_{p}^{2}$ are non-negative, it is natural to identify the lowest state (corresponding to $\gamma_{0}$ ) with the recently discovered Higgs boson, yielding

$$
m_{h}=m_{0}=\sqrt{\frac{25}{4} \operatorname{sech}^{2} k \pi+\gamma_{0}} \frac{k}{R_{y}} a_{\pi} .
$$

Parameterizing the vev $v$ as

$$
v=\frac{\lambda_{v}}{\sqrt{2 \pi}} R_{y}^{-1}=\frac{\lambda_{v}}{\sqrt{2 \pi}} \aleph^{-1} r_{z}^{-1}
$$

where $\lambda_{v} \lesssim 1$, we have

$$
\lambda_{v}=\sqrt{2 \pi\left(\frac{25}{4} \operatorname{sech}^{2} k \pi+\gamma_{0}\right)} \frac{k}{g} \frac{M_{w}}{m_{h}},
$$

a relation that would prove to be useful in identifying the "right" part of the parameter space. It should be remembered, though, that this result is only an indicative one and can receive large corrections as we shall see later.

\subsection{The Higgs spectrum}

The solution to the equation of motion (eq. (3.3)) is given by

$$
\begin{aligned}
\chi_{p}\left(x_{5}\right) & =\operatorname{sech}^{2}(k z)\left[\cot \theta_{p} P_{\nu_{p}^{(h)}}^{3 / 2}\left(\tanh k x_{5}\right)+Q_{\nu_{p}^{(h)}}^{3 / 2}\left(\tanh k x_{5}\right)\right] \\
\nu_{p}^{(h)} & \equiv \frac{-1}{2}+\frac{1}{2} \sqrt{9+4 \gamma_{p} \cosh ^{2}(k \pi)} .
\end{aligned}
$$

Since the solutions have to be even functions of $x_{5}$, we have $\chi_{p}^{\prime}\left(x_{5}=0\right)=0$. Using the identities

$$
\begin{aligned}
& \left(\frac{d P_{N}^{M}(x)}{d x}\right)_{x=0}=\frac{2^{M+1}}{\sqrt{\pi}} \sin \left(\frac{\pi(N+M)}{2}\right) \frac{\Gamma(1+(N+M) / 2)}{\Gamma((N-M+1) / 2)} \\
& \left(\frac{d Q_{N}^{M}(x)}{d x}\right)_{x=0}=2^{M} \sqrt{\pi} \cos \left(\frac{\pi(N+M)}{2}\right) \frac{\Gamma(1+(N+M) / 2)}{\Gamma((N-M+1) / 2)}
\end{aligned}
$$

we are led to

$$
\cot \theta_{p}=\frac{-\pi}{2} \cot \frac{\pi\left(\nu_{p}^{(h)}+3 / 2\right)}{2}
$$

\footnotetext{
${ }^{4}$ This would not have been the case had we not effected the aforementioned scaling of the potential and the $x_{5}$ derivative term.
} 


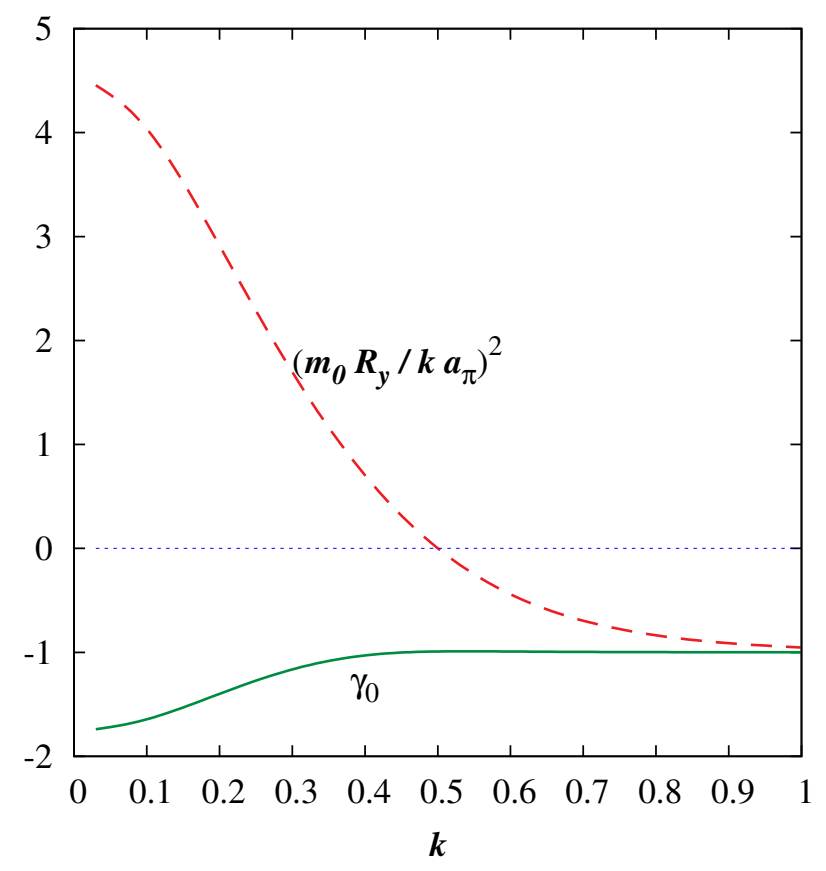

Figure 1. The dependence of the lowest lying Higgs mass on $k$ starting from $\phi_{\mathrm{cl}}=v / \sqrt{r_{z} b\left(x_{5}\right)}$.

Since we are interested only in the small- $k$ branch, the Legendre functions are well-behaved in the entire domain and the use of the Neumann boundary conditions is straightforward, giving rise to

$$
\begin{aligned}
0= & \left(1-2 \nu_{p}^{(h)}\right)\left[\cot \theta_{p} P_{\nu_{p}^{(h)}+1}^{3 / 2}\left(\tau_{\pi}\right)+Q_{\nu_{p}^{(h)}+1}^{3 / 2}\left(\tau_{\pi}\right)\right] \\
& +2\left(\nu_{p}^{(h)}-1\right) \tau_{\pi}\left[\cot \theta_{p} P_{\nu_{p}^{(h)}}^{3 / 2}\left(\tau_{\pi}\right)+Q_{\nu_{p}^{(h)}}^{3 / 2}\left(\tau_{\pi}\right)\right],
\end{aligned}
$$

where $\tau_{\pi} \equiv \tanh (k \pi)$. This equation has to be solved numerically to obtain the discrete set of values allowed to $\nu_{p}^{(h)}$ and, hence, $\gamma_{p}$.

Before we attempt this, it is amusing to note that a negative value for $\gamma_{0}$ would turn $\nu_{0}^{(h)}$ complex. Note though that $\operatorname{Im}\left(P_{\nu_{0}^{(h)}}^{3 / 2}\left(\tanh \left(k x_{5}\right)\right)\right)=\operatorname{Re}\left(Q_{\nu_{0}^{(h)}}^{3 / 2}\left(\tanh \left(k x_{5}\right)\right)\right)=0$, if $\operatorname{Re}\left(\nu_{0}^{(h)}\right)=-1 / 2$, as is the case here. In other words, the boundary conditions demand that, in such cases, $\cot \theta_{p}$ must be a pure imaginary number, as indeed is the case (see eq. (3.9)). The phase of the corresponding wavefunction would, thus, be independent of $x_{5}$.

In figure 1 , we display the result for $\gamma_{0}$ as function of $k$. Also shown, for ready reference, is the dependence of the lowest mass $m_{0}$. As the figure clearly shows, the formulation allows for only $k \lesssim 0.5$. On the other hand, a perusal of table 1 (where the ratio $\aleph$ has been chosen to ensure that $m_{0}$ is consistent with the measured value) shows that requiring $\lambda_{v} \lesssim 1$ (as argued for earlier) would constrain us to $k \gtrsim 0.4$. This, then, seems to put strong constraints on the parameter space. It should be appreciated that the fast growth of the excited state masses with $k$ is but a consequence of the fact that, for such cases, 


\begin{tabular}{|c|c|c|l|}
\hline \multicolumn{4}{|c|}{$k=0.3, \alpha=49.0, w=2.69 \times 10^{-14}$} \\
\hline$(p)$ & $\gamma_{p}$ & $m_{p}(\mathrm{TeV})$ & \\
\hline$(0)$ & -1.16419 & 0.121 & $\lambda_{v}=1.99, \beta=0$ \\
\hline$(1)$ & 4.932 & 0.260 & \\
\hline$(2)$ & 24.742 & 0.489 & \\
\hline
\end{tabular}

\begin{tabular}{|c|c|c|l|}
\hline \multicolumn{4}{|c|}{$k=0.4, \alpha=46.5, w=4.33 \times 10^{-14}$} \\
\cline { 2 - 4 }$(p)$ & $\gamma_{p}$ & $m_{p}(\mathrm{TeV})$ & \\
\hline$(0)$ & -1.029 & 0.120 & $\lambda_{v}=1.71, \beta=0$ \\
\hline$(1)$ & 1.59 & 0.262 & \\
\hline$(2)$ & 9.536 & 0.484 & \\
\hline
\end{tabular}

\begin{tabular}{|c|c|c|l|}
\hline \multicolumn{4}{|c|}{$k=0.5, \alpha=46, w=3.2 \times 10^{-13}$} \\
\hline$(p)$ & $\gamma_{p}$ & $m_{p}(\mathrm{TeV})$ & \\
\hline$(0)$ & -0.9914 & 0.118 & $\lambda_{v}=0.323, \beta=-7 \times 10^{-6}$ \\
\hline$(1)$ & 0.556 & 1.19 & \\
\hline$(2)$ & 3.997 & 2.13 & \\
\hline
\end{tabular}

Table 1. Sample spectra for the small $k$ case for a particular bulk curvature $(\epsilon=0.1)$.

the cancellation between the two pieces in the expression for $m_{0}$ is quite extensive, while this is not the case for the KK states. More interesting is the fact that $k \approx 0.45$ leads to a second scalar state mass of $\sim 700-800 \mathrm{GeV}$ as is indicated in the recent LHC results. As is obvious, the KK-excitation does not acquire a vev, and, hence, has drastically reduced partial width in to a $W W$ or $Z Z$ pair. On the contrary, its coupling with the top-quark (and its KK-cousins) remain unsuppressed, thereby leading to a much larger branching fraction into a $\gamma \gamma$ state. Consequently, it is an obvious candidate to explain the observed excess $[53,54]$. However, it should be realized that there is no conclusive evidence yet for such a resonance, and even less for its angular momentum.

\subsection{Corrections to the Higgs potential and modifications to the spectrum}

That aesthetic considerations (as also phenomenological imperatives as we shall see soon) drive us towards a precipice in the parameter space (as exemplified by a possible tachyonic mode) behoves us to pause and reconsider. Is this a generic feature of the scenario or is it specific to the form of the potential that we have chosen? Even if eq. (3.1) indeed represented the tree-level potential, it would, at the least, be subject to quantum corrections. In fact, given that we are dealing with a non-renormalizable theory (with a well-specified cutoff $R_{y}^{-1}$ ), we could as well consider higher-dimensional terms even in the tree-order Lagrangian. We will, for the sake of simplicity, limit ourselves to polynomial terms.

Even with a generic polynomial modification to the potential, an exact closed-form solution to the equation of motion is not straightforward. Furthermore, the specific form of $\phi_{\mathrm{cl}}$ was chosen to facilitate the solution of the gauge boson wavefunctions with the boundary-localized symmetry breaking term. To this end, we would like to preserve this feature to the best of our abilities and, thus, contemplate only a monomial ${ }^{5}$ perturbation

\footnotetext{
${ }^{5}$ While a polynomial change is but a straightforward generalization of the analysis present here, it adds little to the qualitative features.
} 
to $\phi_{\mathrm{cl}}$ of the form

$$
\phi_{\mathrm{cl}}^{\text {new }}=\frac{v}{\sqrt{r_{z} b(z)}}\left[1+\beta_{n} b^{n}(z)\right]
$$

where $n$ is an as yet undetermined power and $\beta_{n}$ is a small parameter. It is easy to see that the change above can be wrought about with a potential

$$
V_{\text {new }}(\phi)=V(\phi)+\delta V
$$

where

$$
\delta V=-\frac{k^{2}}{R_{y}^{2}} \frac{v}{2 r_{z}^{2}} \beta_{n}\left[\frac{n^{2}+3 n}{n-1}\left(\frac{r_{z} \phi^{2}}{v^{2}}\right)^{1-n}+\frac{n^{2}+2 n+5}{3-n} \operatorname{sech}^{2}(k \pi)\left(\frac{r_{z} \phi^{2}}{v^{2}}\right)^{3-n}\right]+\mathcal{O}\left(\beta_{n}^{2}\right) .
$$

Considerable simplification occurs for $n=-3$ (a choice that we embrace for the rest of the paper), whence the potential simplifies to

$$
\delta V=\frac{-4 k^{2}}{3 R_{y}^{2}} \frac{v}{2 r_{z}^{2}} \beta \operatorname{sech}^{2}(k \pi)\left(\frac{r_{z} \phi^{2}}{v^{2}}\right)^{6}
$$

where $\beta \equiv \beta_{-3} \leq 0$ so as to ensure a potential bounded from below.

Perturbing around $\phi_{\mathrm{cl}}^{\text {new }}$, the new equation of motion is found to be

$$
\frac{1}{R_{y}^{2}} \partial_{5}\left(b^{4} \partial_{5} \hat{\phi}\right)+\frac{b^{2}}{a_{\pi}^{2}} \partial_{\mu} \partial^{\mu} \hat{\phi}=\frac{k^{2}}{R_{y}^{2}}\left[\frac{25 \operatorname{sech}^{2} k \pi}{4} b^{2}-\frac{7}{4} b^{4}+\frac{\beta}{b(z)}\left(25-\frac{28 \aleph}{\lambda_{v}}\right) \operatorname{sech}^{2}(k \pi)\right] \hat{\phi} \text {. }
$$

Treating the last term above as a perturbation, the lowest eigenvalue is shifted to

$$
m_{0, \text { new }}^{2}=\frac{k^{2} a_{\pi}^{2}}{R_{y}^{2}}\left[\left(\frac{25}{4} \operatorname{sech}^{2} k \pi+\gamma_{p}\right)+\beta\left(25-\frac{28}{v r_{z}}\right) X_{k}\right]
$$

where $X_{k}$ is the matrix element of the perturbation Hamiltonian. The consequent shift in the wavefunction $\chi_{0}\left(x_{5}\right)$ can be calculated analogously. For $k=0.5(0.6)$ we have $X_{k}=0.51$ (0.3). Clearly for $\lambda_{v}<1$ (as it should be), a negative $\beta$ raises the Higgs mass considerably, thereby allowing for a wider range of $k$ without risking tachyonic modes. In the new description eq. (3.7) will get modified and, as figure 2 shows, a rather wide range of $\lambda_{v}$ becomes allowed once even small perturbations are switched on.

\subsection{An alternative scheme}

Appealing to corrections to the Higgs potential is not the only way out of the tachyonic imbroglio. We briefly consider, here, an alternative. Recall that the very establishment of the nested warping structure required a $x_{5}$-dependent tension on the 4 -brane at $x_{4}=\pi$ given by

$$
V_{2}\left(x_{5}\right)=-8 M_{6}^{2} \sqrt{\frac{-\Lambda_{6}}{10}} \operatorname{sech}\left(k x_{5}\right)=-8 \epsilon M_{6}^{5} \operatorname{sech}\left(k x_{5}\right) .
$$

The particular form for $V_{2}\left(x_{5}\right)$ could have originated from a variety of mechanisms including a $x_{5}$-dependent vacuum structure in a scalar field theory [48]. As can be appreciated, this 


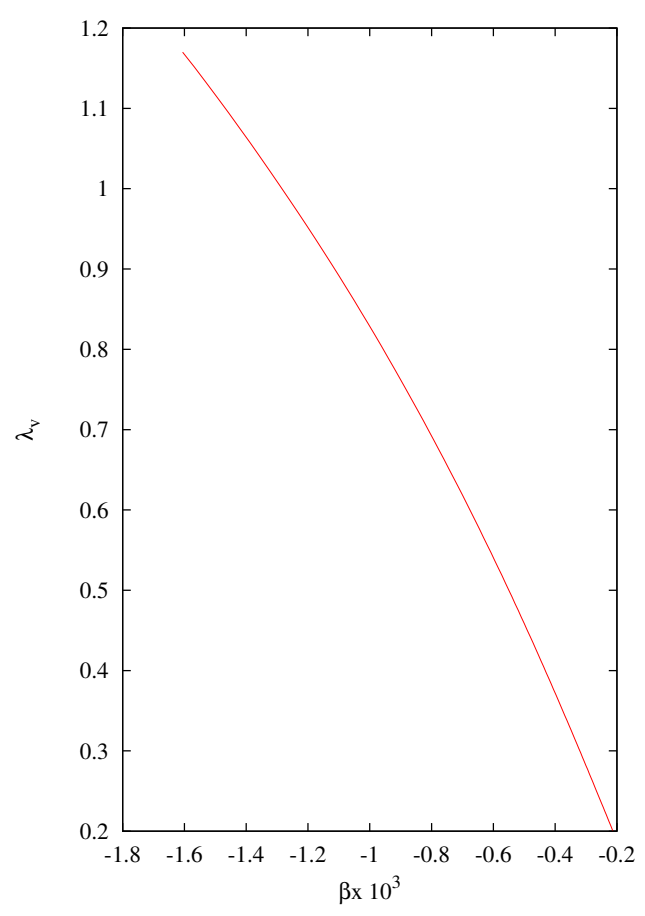

Figure 2. The relation between the parameters $\lambda_{v}$ and the coefficient $\beta_{-3}$ in the monomial scalar field perturbation $\left(\phi^{12}\right)$ that would lead to a light Higgs mass of $125 \mathrm{GeV}$ for $k=0.6$ and $50<\aleph<53$.

is intimately connected to the very process of compactification in this theory. It is, thus, conceivable that such a dynamical system (whatever be the exact mechanism) could couple to the scalar $\phi$ as well. Thus we may posit a scalar field Lagrangian of the form

$$
\widehat{\mathcal{L}}_{\phi}=\delta\left(x_{4}-\pi\right) \sqrt{-g_{5}}\left[\frac{-\gamma V_{2}\left(x_{5}\right)}{M_{6}^{5}}\left\{g^{\mu \nu} D_{\mu} \phi^{\dagger}\left(x^{\bar{M}}\right) D_{\nu} \phi\left(x^{\bar{M}}\right)+R_{y}^{-2}\left|D_{5} \phi\left(x^{\bar{M}}\right)\right|^{2}\right\}+\widehat{V}(\phi)\right]
$$

where $\gamma$ is a dimensionless positive constant. Choosing a standard form for $\widehat{V}(\phi)$, namely

$$
\widehat{V}(\phi)=-\mu^{2} \phi^{\dagger} \phi+\frac{\widehat{\lambda}}{2}\left(\phi^{\dagger} \phi\right)^{2},
$$

would lead to a flat (i.e., $x_{5}$-independent) classical configuration viz. $\widehat{\phi}_{\mathrm{cl}}=\widehat{v}=\sqrt{-\mu^{2} / \widehat{\lambda}}$. The corresponding localized mass term for the gauge field is exactly what we get for the unperturbed potential $V(\phi)$ discussed earlier. On the other hand, with the scalar field $\phi$ now settling to its global minimum, and with the $x_{5}$-dependence of the fluctuation $\hat{\phi}$ (around $\widehat{\phi}_{\mathrm{cl}}=\widehat{v}$ ) only adding to the energy, no tachyonic modes exist any longer. This allows us to use a much wider range of $k$.

It should be realized that these results are not tied to the exact form of $\widehat{\mathcal{L}}_{\phi}$, but would be replicated, to a great extent, for many other choices (for both the kinetic and the potential terms). This fact, as well as the results drawn, in the preceding section, from perturbing the potential leads us to the inescapable conclusion that eq. (3.7) is not an exact relation but only an indicative one. This does not come as surprise, for once radiative 
corrections (whether in the full theory, or in the effective four-dimensional version) to the Higgs potential are taken into account, tree-level relationships would indeed change (as happens, for example in the well-known case of the minimal supersymmetric standard model).

\section{Effective Lagrangian}

To examine the low-energy consequences of this model, and especially those of the electroweak symmetry breaking mechanism, it is useful to construct an effective Lagrangian, which we do now. As we have already seen, the resolution of the hierarchy problem with a localized (whether on a 3-brane or a 4-brane, as done here) Higgs boson introduces nontrivial alterations to the boundary conditions of the gauge bosons. In particular, such a localized energy density deforms not only the mass spectrum, but also the wavefunctions of the KK-modes. However, since the symmetry-breaking mass is much smaller than the KK-masses, it is safe to consider the changes in the lowest (zero-) mode alone, while neglecting those to the others. Moreover, as we shall soon see, the effect of such changes in the KK-mode wavefunctions on low-energy observables are further suppressed.

As we have already learnt, in the absence of the Higgs vev, the zero-mode wavefunctions for the gauge boson, viz. $\chi_{0}\left(x_{5}\right)$ and $\eta_{0,0}\left(x_{4}\right)$, are both flat. On inclusion of the 4-brane localized vev $\phi_{\mathrm{cl}}\left(x_{5}\right)=v / \sqrt{r_{z} b\left(x_{5}\right)}$, the latter changes to $\left(V \equiv W^{ \pm} / Z\right)$

$$
\eta_{0,0}^{V} \rightarrow \eta_{0,0}^{V r} \approx \frac{1}{\sqrt{\pi}}\left[1+\frac{M_{V}^{2} \rho^{2}}{4}\left(e^{2 c\left(x_{4}-\pi\right)}-1-2 c x_{4} e^{2 c\left(x_{4}-\pi\right)}+2 c \pi\right)\right] .
$$

where

$$
\rho=\frac{R_{y}}{c} e^{c \pi}
$$

and we are working under the approximation that $m_{0,0} R_{y} / c \ll 1$.

Before we use eq. (4.1) to calculate any observables, we should also consider changes wrought by the inclusion of the perturbation of eq. (3.13) that would have led to a change in the classical configuration encapsulated in eq. (3.11). The effect of this change in the boundary-localized energy density for the massive gauge bosons can be calculated easily in perturbation theory, and, to the first order in $\beta$, the zero-mode wavefunction changes to

$$
\eta_{0,0}^{V r}\left(x_{4}\right) \rightarrow \eta_{0,0}^{V r}\left(x_{4}\right)+\left[\frac{2 \beta_{n} v^{2} a_{\pi}^{2}}{\pi\left(m_{z}^{2}-M_{(1,0)}^{2}\right)} \int_{-\pi}^{\pi} d x_{5} b^{n+1}\left(x_{5}\right)\left[\chi_{0}\left(x_{5}\right)\right]^{2}\right] \eta_{1,0}^{V r}\left(x_{4}\right)+\cdots
$$

where the ellipsis denote the sub-dominant terms. With the integral being $\lesssim \mathcal{O}(1)$, the additional suppression of $v^{2} / M_{(1,0)}^{2}$ renders this correction too small to be of any interest, and we shall neglect it altogether henceforth.

Reverting to eq. (4.1), such distortions manifest themselves, on integrating out the extra dimensions, as wavefunction renormalizations. On canonically normalizing the kinetic term in the Lagrangian, this brings forth tree level modification in the gauge mass term as also any gauge interaction terms. The relevant part of the renormalized Lagrangian, 
for the renormalized zero mode $V^{r}$, can be written in terms of the self energy corrections $\Pi_{V V}\left(q^{2}\right)$ as

$$
\mathcal{L}_{V}=-\frac{1}{4} \mathcal{Z} F^{r \mu \nu} F_{\mu \nu}^{r}-\frac{1}{2}\left[M_{V}^{2}+\Pi_{V V}(0)\right] V^{r 2},
$$

where $M_{V}$ arises from the Higgs vev and

$$
\begin{aligned}
\Pi_{V V} & =\left\{\int_{0}^{\pi} d x_{4} a^{2}\left(x_{4}\right)\left[\partial_{4} \eta_{0,0}^{V r}\left(x_{4}\right)\right]^{2}\right\}\left\{\int_{0}^{\pi} d x_{4}\left[\eta_{0,0}^{V}\left(x_{4}\right)\right]^{2}\right\}^{-1} \\
\mathcal{Z} & =1-\Pi_{V V}^{\prime}(0)=\left\{\int_{0}^{\pi} d x_{4}\left[\eta_{0,0}^{V r}\left(x_{4}\right)\right]^{2}\right\}\left\{\int_{0}^{\pi} d x_{4}\left[\eta_{0,0}^{V}\left(x_{4}\right)\right]^{2}\right\}^{-1} .
\end{aligned}
$$

Here, $\Pi_{V V}^{\prime} \equiv \partial_{q^{2}} \Pi_{V V}$. Note that, since the wave function in the $x_{5}$-direction remains constant and unchanged, there is no corresponding contribution to $\Pi_{V V}$ or $\Pi_{V V}^{\prime}$.

Post electroweak symmetry breaking, we are primarily interested in the lowest modes, and the relevant part of the mass matrix can be diagonalized by a transformation analogous to that in the SM, viz.

$$
Z_{\mu}=c_{\theta} W_{\mu}^{3}-s_{\theta} B_{\mu}, \quad A_{\mu}=s_{\theta} W_{\mu}^{3}+c_{\theta} B_{\mu}, \quad c_{\theta} \equiv \frac{g}{\sqrt{g^{2}+g^{\prime 2}}} .
$$

Here, we neglect the small mixing with the higher KK-levels, which constitutes an excellent approximation. In the basis where the mass matrix is diagonal, the relevant part of the Lagrangian could be written as

$$
\begin{aligned}
-\mathcal{L}_{\text {eff }}= & \frac{\mathcal{Z}_{\gamma}}{4} F_{\mu \nu} F^{\mu \nu}+\frac{\mathcal{Z}_{W}}{2} W_{\mu \nu}^{+} W^{-\mu \nu}+\frac{\mathcal{Z}_{Z}}{4} Z_{\mu \nu} Z^{\mu \nu} \\
& +\left[M_{w}^{2}+\Pi_{w w}(0)\right] W_{\mu} W^{\mu}+\frac{1}{2}\left[M_{z}^{2}+\Pi_{z z}(0)\right] Z_{\mu} Z^{\mu} \\
\equiv & \frac{1+A}{4} F_{\mu \nu} F^{\mu \nu}+\frac{1+B}{2} W_{\mu \nu}^{+} W^{-\mu \nu}+\frac{1+C}{4} Z_{\mu \nu} Z^{\mu \nu}+\frac{G}{2} F_{\mu \nu} Z^{\mu \nu} \\
& +(1+w) M_{W}^{2} W_{\mu} W^{\mu}+\frac{1+z}{2} M_{z}^{2} Z_{\mu} Z^{\mu}
\end{aligned}
$$

where we have deliberately introduced the parameters $A, B, C, G, w, z$ for future ease.

Since the photon does not couple to the Higgs, $\Pi_{\gamma \gamma}=0$ and the corresponding renormalization factor $\mathcal{Z}_{\gamma}=1$. For the $W$ and $Z$, we get instead

$$
\begin{aligned}
\Pi_{w w}(0) & =\frac{1}{2} M_{w}^{2}\left(M_{w} \rho\right)^{2}\left(\frac{1}{2 c \pi}-1+c \pi\right) \equiv \frac{1}{2} M_{w}^{2} \tilde{m}_{w} \\
\Pi_{z z}(0) & =\frac{1}{2} M_{z}^{2}\left(M_{z} \rho\right)^{2}\left(\frac{1}{2 c \pi}-1+c \pi\right) \equiv \frac{1}{2} M_{z}^{2} \tilde{m}_{z}
\end{aligned}
$$

which also implies that

$$
\begin{aligned}
\mathcal{Z}_{w} & =1-\Pi_{w w}^{\prime}=1-g^{2} \Pi_{11}^{\prime} \approx 1+\tilde{m}_{w} \\
\mathcal{Z}_{z} & =1-\Pi_{z z}^{\prime}=1-\left(g^{2}+g^{\prime 2}\right) \Pi_{33}^{\prime} \approx 1+\tilde{m}_{z} .
\end{aligned}
$$


With the rest of the gauge sector unchanged, the gauge-fermion interaction can now be expressed in the standard form, viz.

$\mathcal{L}_{\text {int }}=\left[g \sum_{i, j} V_{i j} \bar{\psi}_{i} \gamma^{\mu} P_{L} \psi_{j} W_{\mu}^{+}+\right.$H.c. $]+\frac{g}{c_{\theta}} \sum_{i} \bar{\psi}_{i} \gamma^{\mu}\left(T_{3 i} P_{L}-Q_{i} s_{\theta}^{2}\right) \psi_{i} Z_{\mu}+e \sum_{i} \bar{\psi}_{i} \gamma^{\mu} Q_{i} \psi_{i} A_{\mu}$,

with all modifications encoded in the aforementioned six parameters $A, B, C, G, w$ and $z$. Note, though, that, on redefining $W_{\mu}^{a}, B_{\mu}$ and Higgs fields, only three of the six would remain independent and have been famously parametrized as $S, T, U$ [56] (or, equivalently, $\left.\epsilon_{1,2,3}[57]\right)$ through the relations

$$
\begin{aligned}
\alpha_{\mathrm{em}} S & \equiv 4 s_{\theta}^{2} c_{\theta}^{2}\left[A-C-\frac{c_{\theta}^{2}-s_{\theta}^{2}}{s_{\theta} c_{\theta}} G\right] \\
\alpha_{\mathrm{em}} T & \equiv w-z \\
\alpha_{\mathrm{em}} U & \equiv 4 s_{\theta}^{2}\left[A-\frac{B}{s_{\theta}^{2}}+\frac{c_{\theta}^{2}}{s_{\theta}^{2}} C-2 \frac{c_{\theta}}{s_{\theta}} G\right] .
\end{aligned}
$$

\subsection{The oblique parameters}

Having considered the general form, we now concentrate on the particular case at hand, namely the extra corrections wrought by the new physics over and above the SM contributions, with the latter accruing only at the loop level. On the contrary, the additional contributions here are two-fold. One set is occasioned by the exchange of the KK-excitations and these we shall come back to later. The other is occasioned by a change in the wavefunctions of the SM particles and appear even at the tree-level. Given this, we may as well neglect any loop-level effects associated with the new physics. This approximation immediately leads to certain simplifications. For example, consider $\Pi_{3 Q}$, which, in the SM, is generated only at the loop-level. Since ours is a tree-level calculation of the new physics effect, no additional $Z-\gamma$ mixing can be induced $\left(\delta \Pi_{3 Q}=0\right)$. This, of course, was evident from eq. (4.3) as it implied $G=0$.

Renormalizing the fields through

$$
A_{\mu} \rightarrow A_{\mu}^{r}=A_{\mu}, \quad Z_{\mu} \rightarrow Z_{\mu}^{r}=\sqrt{\mathcal{Z}_{z}} Z_{\mu}, \quad W_{\mu} \rightarrow W_{\mu}^{r}=\sqrt{\mathcal{Z}_{w}} W_{\mu}
$$

the gauge kinetic term can be expressed as

$$
\mathcal{L}_{\mathrm{eff}}=-\frac{1}{4} F_{\mu \nu}^{r} F^{r \mu \nu}-\frac{1}{2} W_{\mu \nu}^{r+} W^{r-\mu \nu}-\frac{1}{4} Z_{\mu \nu}^{r} Z^{r \mu \nu}+M_{w}^{r 2} W^{r 2}+\frac{1}{2} M_{w}^{r 2} Z^{r 2},
$$

where the renormalized masses are given by

$$
\begin{aligned}
& M_{w}^{r 2}=M_{w}^{2}(1+w-B)=M_{w}^{2}\left[1-\frac{1}{2} \tilde{m}_{w}\right] \\
& M_{z}^{r 2}=M_{z}^{2}(1+z-C)=M_{z}^{2}\left[1-\frac{1}{2} \tilde{m}_{z}\right] .
\end{aligned}
$$


Similarly, the gauge fermion interaction is given by

$$
\begin{aligned}
\mathcal{L}_{\text {int }}= & {\left[\frac{g}{\sqrt{\mathcal{Z}_{w}}} \sum_{i j} V_{i j} \bar{\psi}_{i} \gamma^{\mu} P_{L} \psi_{j} W_{\mu}^{r+}+\text { H.c. }\right] } \\
& +\frac{g}{c_{\theta} \sqrt{\mathcal{Z}_{z}}} \sum_{i} \bar{\psi}_{i} \gamma^{\mu}\left(T_{3 i} P_{L}-Q_{i} s_{\theta}^{2}\right) \psi_{i} Z_{\mu}^{r}+e \sum_{i} \bar{\psi}_{i} \gamma^{\mu} Q_{i} \psi_{i} A_{\mu}^{r} .
\end{aligned}
$$

This immediately leads to expressions for the oblique parameters

$$
\begin{aligned}
\delta S & \approx-4 \pi \frac{M_{w}^{2} \rho^{2} c \pi}{g^{2}}=-4 \pi \zeta \\
\delta T & \approx \frac{-\pi}{2 \cos ^{2} \theta_{w}} \frac{M_{w}^{2} \rho^{2} c \pi}{g^{2}}=\frac{-\pi}{2 \cos ^{2} \theta_{w}} \zeta \\
\delta U & =0 \\
\zeta & \equiv \frac{M_{w}^{2} \rho^{2} c \pi}{g^{2}} .
\end{aligned}
$$

A detailed fit to the data has been performed in [58], and we use their central values (derived by fixing $U=0$, as is the case here and as is normal for most beyond-SM fits) of $S=0.00 \pm 0.08$ and $T=0.05 \pm 0.07$.

\section{$4.2 G_{f}$}

In the most popular renditions of the SM fields leaking into a flat bulk (the so-called Universal Extra Dimension scenarios), the existence of a $Z_{2}$ symmetry prevents the odd KK-modes of the gauge bosons from coupling with the SM bilinear. Furthermore, the couplings of the even-modes are progressively suppressed for the higher modes. No such symmetry exists here, and all modes of the gauge-bosons would couple with non vanishing strengths to the zero-mode fermion bilinear. In particular, the coupling of the $(1,0)$-mode is often enhanced with respect to the SM coupling. This immediately leads to a change in the four-fermion operators. For charged current processes at low energies, this is parametrized by the very well measured quantity $G_{f}$ which now reads

$$
G_{f}=G_{f}^{\mathrm{SM}}\left[1+\sum_{(n, p) \neq(0,0)}\left(\frac{g^{(n, p)} M_{W}}{g M_{W(n, p)}}\right)^{2}\right] \approx G_{f}^{\mathrm{SM}}\left[1+\left(\frac{g^{(1,0)} M_{W}}{g M_{W(1,0)}}\right)^{2}\right]
$$

To appreciate the approximation above, it should be remembered that, for a given $p$, it is the coupling of the $n=1$ mode, viz. $g^{(1, p)}$, that is the largest, while those for the higher $n$-modes are, typically, somewhat suppressed with respect to the SM coupling (see table 1 of ref. [55]). Compounded by the fact that the higher modes are much heavier, it is clear that, within the $p=0$ tower, the contribution of the $n=1$ mode dominates. For $p \neq 1$ modes, all the couplings are significantly suppressed (even for $n=0$ ) and the masses larger. 


\begin{tabular}{|c|c|c|c|}
\hline \multicolumn{3}{|c|}{$k=0.5, \alpha=48.367, w=7.081 \times 10^{-14}$} \\
\hline$(n, p)$ & $m_{n p}(\mathrm{TeV})$ & $C_{n p}$ & $V$ \\
\hline$(1,0)$ & 9.5 & 3.81 & $1.0 \times 10^{-3}$ \\
\hline$(2,0)$ & 21.9 & 0.49 & $3.34 \times 10^{-6}$ \\
\hline$(0,1)$ & 17.0 & 0.20 & $9.21 \times 10^{-7}$ \\
\hline$(1,1)$ & 30.9 & 0.06 & $2.84 \times 10^{-8}$ \\
\hline
\end{tabular}

Table 2. Sample spectrum for the small $k$ case for a particular bulk curvature $(\epsilon=0.1)$ and with $\lambda_{v}=1.5 . C_{n p}$ is defined as the ratio of $g^{(n, p)}$ and $g$.

In other words, we have

$$
G_{f} \approx G_{f}^{\mathrm{SM}}[1+V], \quad V \equiv \frac{\zeta}{\pi c}\left(\frac{g^{(1,0)}}{x_{1,0}}\right)^{2} .
$$

Experiments demand [11] $V<0.0013$ at 95\% C.L.

To a reasonable degree of accuracy, the coupling of the $W^{ \pm(1,0)}$ to the fermion bilinears could be approximated as $g^{(1,0)} \sim 3.8 \times g$. Using this, we have $M_{W^{(1,0)}} \gtrsim 8.6 \mathrm{TeV}$, a constraint that is a little weaker than that operative for the RS case. This was not unexpected, because the suppression of the gauge-excitation coupling (in relation the five-dimensional analogue), is only a small one, as evinced by the aforementioned approximation. What is more interesting is that, as table 2 shows, there exists a large parameter space where this constraint is automatically satisfied. We will delineate this quantitatively in the next section.

\section{Confronting electroweak precision measurements}

Rather than drawing conclusions piecemeal from individual data (as we have done in the preceding section), we now attempt to examine how well the model agrees globally with all the precision measurements. Ref. [14] drew up expressions for 22 such observables in terms of the their SM values, the oblique parameters [56] $S, T, U$ and $V$ (the shift in $G_{F}$ ). While the extra-dimensional contributions to the $U$-parameter are vanishingly small, for $S, T$ and $V$, we use the expressions derived in the preceding section. Re-evaluating the $\mathrm{SM}$ expectations for a $125 \mathrm{GeV}$ Higgs, ${ }^{6}$ we may now construct a $\chi^{2}$-test for this model comparing the expressions with the experimental results [58].

While one could attempt a multidimensional analysis optimizing all the parameters in the theory, it is much more instructive to examine the dependence of the $\Delta \chi^{2}$ (the shift in the $\chi^{2}$ from the SM value of $\approx 27.5$ ) on individual parameters. To this end, we must first identify the appropriate set of independent parameters, and the range that they may

\footnotetext{
${ }^{6}$ More up-to-date analyses, including two-loop results, are available [59-61], but make little qualitative difference to our conclusions.
} 

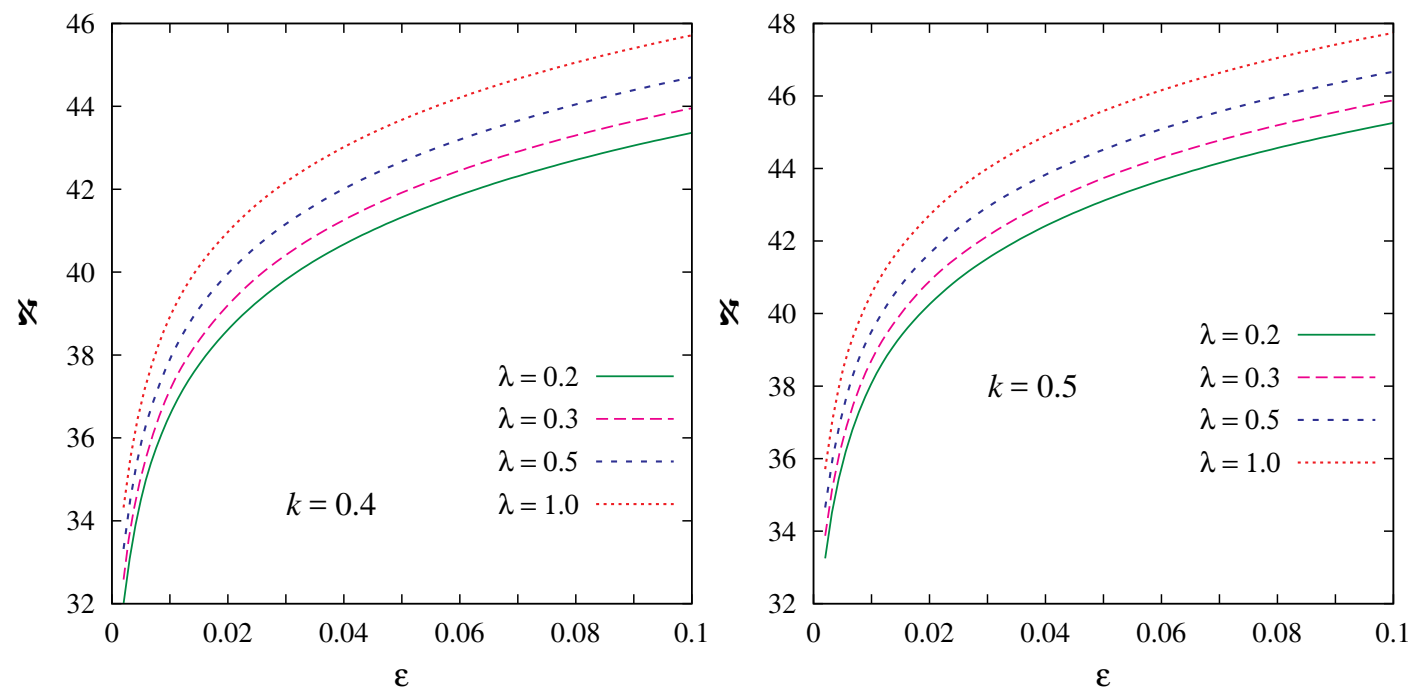

Figure 3. The relation between the parameters $\epsilon$ and $\aleph$ that reproduces the electroweak breaking scale correctly. The left and right panels refer to $k=0.4$ and $k=0.5$ respectively.

be allowed. To start with, eq. (2.2) imposes two independent relations between $c, \aleph, k, \epsilon$ and the product $r_{z} M_{6}$. Now, the applicability of a semi-classical treatment of the gravity sector requires that the curvature be sufficiently smaller than the fundamental scale $M_{6}$, or in other words, $\epsilon \lesssim 0.1$. Similarly, the avoidance of a large hierarchy implies that the product $M_{6} r_{z}$ be not too large. Since we are interested in the small $k$ regime $(k \lesssim 1)$, this immediately puts a lower bound on $\epsilon$. A complementary relation is provided by eq. (3.6), and, once the electroweak scale is specified, the resultant relation between the parameters is determined as displayed in figure 3. As is apparent, the dependence on $k$ is minimal, owing to the fact that the function $k / \cosh (k \pi)$ is slowly varying in the region of interest.

In figure 4 , we present the corresponding shifts $\Delta \chi^{2}$. Understandably, the dependence on $k$, once again, is minimal. As eq. (3.6) shows, a smaller $\lambda_{v}$ would imply a larger $R_{y}^{-1}$. This, in turn, has two consequences. First, it implies larger masses for the KK-excitations of the gauge boson masses and, hence, a smaller change to $G_{f}$. Simultaneously, it results in smaller values for $\rho$ (see eq. (4.2)), and, hence, smaller values for both $\delta S$ and $\delta U$. Thus, it is easy to understand the dependence of $\Delta \chi^{2}$ on $\lambda_{v}$. It is interesting to note that even a very moderate hierarchy $\left(\lambda_{v} \lesssim 0.3\right)$ renders the model quite consistent with low-energy data, whereas $\lambda_{v} \sim 0.2$ makes it almost indistinguishable from the SM.

\section{Beta function}

Grand unification remains a holy grail for scenarios of physics beyond the SM, for not only does it provide a unification of forces, but also a platform to answer questions pertaining to inflation and baryogenesis on the one hand, and a formalism to understand fermion masses on the other. Within the standard four-dimensional paradigm, gauge coupling unification occurs, though only at scales in the vicinity of $10^{15}-10^{16} \mathrm{GeV}$, thereby putting a direct verification of the paradigm beyond the reach of experiments in the foreseeable future. 

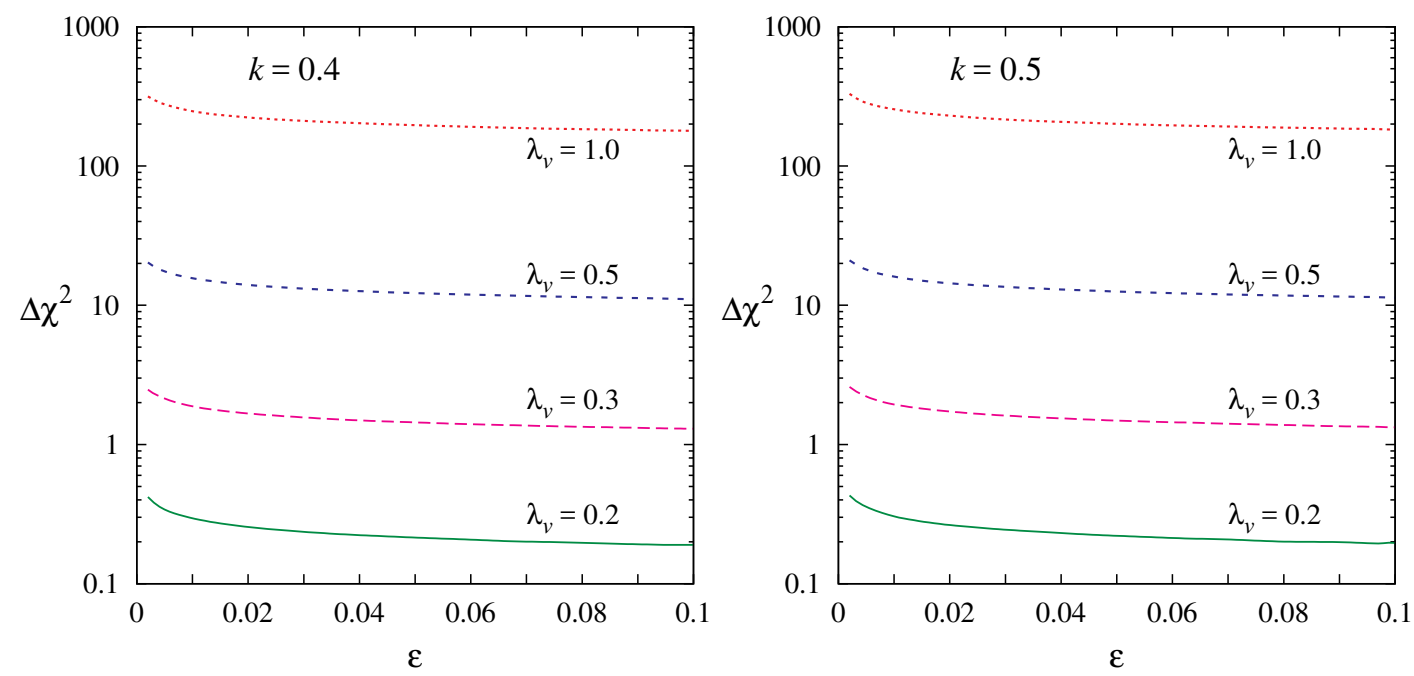

Figure 4. The shift in the $\chi^{2}$ (as derived from the 22 observables listed in ref. [14]) as a function of $\epsilon$ for various values of the parameter $\lambda_{v}$. The left and right panels refer to $k=0.4$ and $k=0.5$ respectively, while the parameter $\aleph$ has been fixed as in figure 3 .

A curious thing happens in the case of the universal extra-dimensional scenarios. The renormalization group evolution of the gauge couplings (which is logarithmic in the case of the SM) now turns power-law [63, 64]. This can be understood most easily in terms of the KK-reduction, whereby the logarithmic contributions from each of the individual KK-excitations sum up to give a power-law behaviour. ${ }^{7}$

For warped geometries, as is the case here, additional features arise. The absence of a KK-parity implies the existence of additional loops. A further complication is caused by the fact that, owing to the nontrivial differences in their wavefunctions, distinct KK-levels of the same field have differing coupling strengths. This already renders the evolution to be quite different from the UED case.

And, finally, there is the issue of the graviton loops. Unlike in the UED case, here the couplings of the KK-gravitons are non-negligible and ought to be included. On the other hand, such a inclusion cannot be made in a straightforward fashion for the entire treatment of the gravitation sector has been semi-classical and loop calculations with gravitons are ill-defined.

In view of this, we desist from considering any graviton-loops. This can also be justified in the sense, that for a given KK-level, the graviton is not only heavier than the SM excitations, but also has a effective coupling ${ }^{8}$ significantly smaller than them. Naively at least, the graviton contributions to the gauge beta-functions would, thus, be expected to be numerically small. Hence, while our results cannot be termed exact, they are expected to be very good approximations of calculations in the full theory.

\footnotetext{
${ }^{7}$ Much the same would be seen if the entire calculation were to be done in the full five-dimensional theory. Care must be taken, though, in view of the inherently non-renormalizable nature of the theory.

${ }^{8}$ The graviton coupling is, of course, dimensionful. What should be compared to the effective $g_{\mathrm{YM}}$ is the product of the graviton mass and its coupling. In the small $k$ regime, this is indeed much smaller [51, 52].
} 

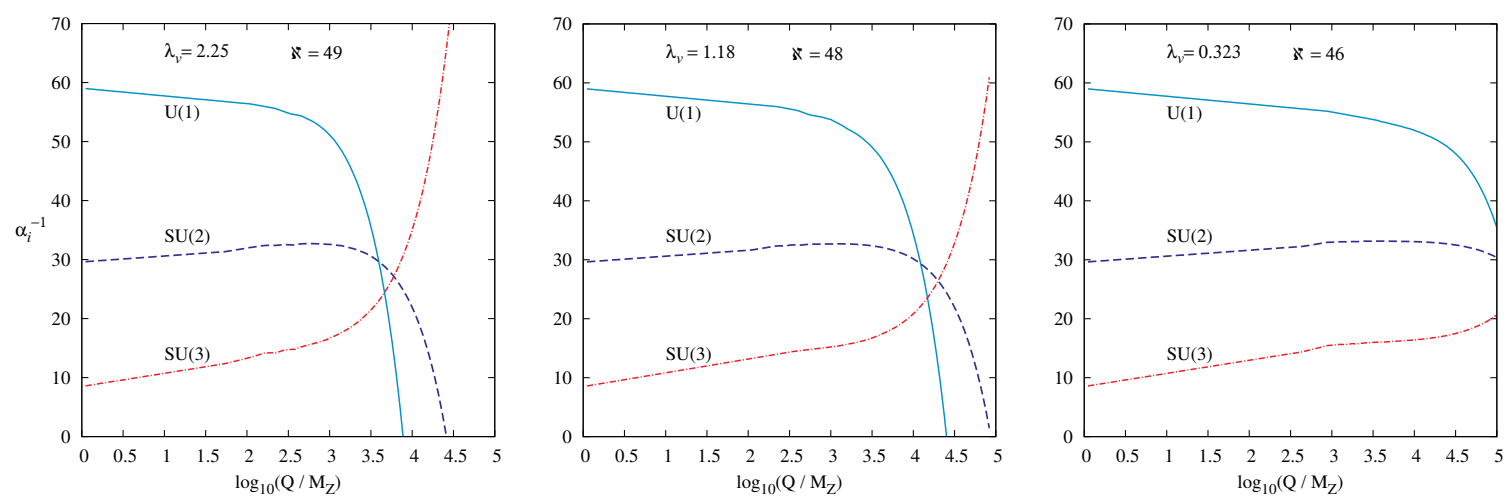

Figure 5. Gauge coupling constant evolution for $k=0.5, \epsilon=0.1$. The three panels correspond to different $\lambda_{v}$ values.

Restricting ourselves to a discussion of the interactions between the SM fields, the one-loop $\beta$-functions can be calculated in a straightforward manner, considering the KKexcitations to be heavy particles with appropriate couplings (gauge, Yukawa) with the corresponding contributions to be included as a threshold is crossed..$^{9}$

As already mentioned, even this task is rendered difficult by the fact that KK-number, or even KK-parity, is not conserved. However, as shown in [55], as far as the interactions of the zero mode gauge fields are concerned, KK-number is indeed conserved. This is exactly true as long as the modifications due to Higgs localization can be neglected, which it can indeed be above the electroweak symmetry breaking scale. The change in evolution of the hypercharge is straightforward as we only need to calculate the additional contributions to the vacuum polarization. Similarly, for the non-abelian component of the theory, the task, at one-loop order, is easier for the triple- "gluon" vertex ${ }^{10}$ (for all the vertices now respect KK-number conservation) than for the gauge-fermion vertex. This can be exploited, in conjunction with the appropriate Slavnov-Taylor identities (since our six-dimensional Lagrangian is gauge invariant) to calculate the RG flow for the other vertices as well. Thus, the exercise is very similar to that in the universal extra-dimension scenarios, but for the added complication of unevenly placed KK-masses that need to be calculated numerically. ${ }^{11}$ It should be appreciated that the same results are obtained for vertices that admit KK non-conservation, but only if all the modes are taken into account.

In figure 5, we display the evolution of the gauge coupling constants for a particular parameter point, namely $(k=0.5, \epsilon=0.1)$ and some representative values of $\lambda_{v}$. As is expected, the evolution is indeed much faster than in the SM, and the "unification" scale is lowered to approximately $\approx 10^{3}-10^{6} \mathrm{TeV}$. The lower $\lambda_{v}$ is, the higher are the masses for the KK-excitations, and, consequently, the higher is the unification scale. On the other hand, if we introduce a mechanism (such as those including a custodial symmetry) that allows us to significantly lower the KK-masses, the unification scale would be lowered instead.

\footnotetext{
${ }^{9}$ Since we are effecting only a one-loop calculations, neglecting the threshold effects is an excellent approximation.

${ }^{10}$ The same holds for the four-"gluon" vertex as well, except that more diagrams need to be calculated.

${ }^{11}$ In actuality, after the first few levels, the rest can be rather well-fitted in terms of a bilinear function.
} 
Two additional features are worth commenting on. The first is that, not only the $\mathrm{U}(1)_{Y}$ theory, but also the $\mathrm{SU}(2)_{L}$ theory lacks asymptotic freedom, a consequence of the number of new states in the theory. This is quite analogous to the case of the UED [64]. A related feature is the presence of some small kinks in the plots, visible most prominently for the case of the $\mathrm{SU}(3)$. This, once agaian, is but reflective of momentary change of the sign of the $\beta$-function and owes its origin to the relative placements of the KK-excitations. Note that the latter feature is particularly sensitive to the order to which the RG-equations are calculated, and stand to be significantly altered once we go beyond treating thresholds as discrete steps. Furthermore, such effects could also play a role in resolving the lack of exact unification.

\section{Summary and outlook}

While a five-dimensional world with a warped metric and the SM fields confined on a end-of-the-world brane (the RS scenario) offered a tantalizing solution to the hierarchy problem, it suffers from the obvious problem that no KK-excitation of the graviton has been observed so far. Similarly, if one were to calculate amplitudes for flavour-changing neutral currents, the low cut-off $(\sim 1 \mathrm{TeV})$ of the theory implies that the dimension-six operators do not suffer a large suppression and the resultant rates are too high. The first problem can be solved [51,52] courtesy reduced couplings of the gravitons in a sixdimensional generalization [48] of the original RS model with nested warping. Indeed, the coupling can be suppressed well enough for the recently reported diphoton excess at $750 \mathrm{GeV}[53,54]$ to be explained in terms of such a resonance [52], a feat impossible within the five-dimensional paradigm.

Allowing the fermions and gauge fields to propagate in the bulk is an obvious antidote to the second problem, since four-Fermi operators are now suppressed by higher powers of the ultraviolet cutoff. On the other hand, doing so will bring into play KK-towers of the fermions and gauge bosons and these, in turn, will effect low-energy observables thereby inviting tight constraints from the indirect measurement data obtained at LEP. Indeed, within the five-dimensional paradigm, such constraints push the gauge boson KK-masses well beyond the reach of the LHC [12-14]. This brings back at least a little hierarchy unless additional physics such as new particles alongwith a custodial symmetry is invoked.

Clearly, both sets of problems could be addressed if one considers bulk gauge bosons and fermions in a six-dimensional theory with nested warpings, and the required formalism was introduced in Paper I [55]. Such a construction brings forth several interesting consequences such as restrictions on the number of chiral generations. Furthermore, with one particular tower disappearing identically for each fermion species, if such KK-fermions (KK-bosons) can be produced at a collider, the signatures would be quite non-canonical. In the current paper, we examine the issue of electroweak symmetry breaking in this scenario as well as consider the phenomenological implications and constraints.

Contrary to the case of the fermions and gauge bosons, the Higgs cannot percolate into the six-dimensional bulk, for it would bring back the hierarchy problem. While it might seem that confining it to a 3-brane would be the simplest solution, this, unfortunately 
presents some technical complications (as discussed in Paper I). Instead, we consider a novel mechanism confining it to a 4-brane, with the Higgs acquiring a $x_{5}$-dependent classical configuration thanks to an interplay between the potential term and the nontrivial kinetic term endemic to a curved background. The maximum $v$ of the classical configuration is naturally of the order of the cutoff $R_{y}^{-1}$ (suffering only a mild suppression $0.1 \lesssim \lambda_{v} \lesssim 1$ ), but is warped down to the electroweak scale. Interestingly, the simplest such construction puts a limit $k \lesssim 0.5$, beyond which tachyonic modes develop. The five-dimensional nature of the Higgs field is manifested in the shape of KK-resonances, which often tend to be quite light if tree level relations to equate the zero-mode mass to $125 \mathrm{GeV}$. However, once quantum corrections are included, the KK-masses are lifted considerably (alongwith significantly relaxing the constraint on the parameter $k$ ). For example, for $k=0.4$ and $\lambda_{v}=0.5$, a small perturbation $\beta_{-3} \approx 3.4 \times 10^{-4}$ leads to a first excited mass $M_{h^{(1)}} \simeq 800 \mathrm{GeV}$. This would be of particular interest if the recently reported excess $[53,54]$ in the diphoton channel is actually confirmed. For while the graviton sector can also have such a resonance [52], allowing the SM fields into the bulk not only forces us to a part of the parameter space that increases the mass of the first graviton resonance, but also drastically suppresses thereby reducing the signal strength. Furthermore, if the resonance is to be a graviton, then we should soon see excesses in other channels as well (although the present data is inconclusive). On the other hand, the aforementioned Higgs resonance would not decay to $W / Z$-pairs through tree-level couplings, and with the couplings to the top-sector also being modified considerably, it could present an interesting alternative.

Of more immediate concern are the effects on low-energy phenomenology. With flavour changing neutral current operators now being suppressed by four powers of the UV-cutoff (in contrast with only three powers for the analogous five-dimensional theory), the constraints from this sector are minimal. On the other hand, the very confinement of the Higgs onto a 4-brane introduces changes in the gauge-boson wavefunctions that manifest themselves in the form of additional tree-level contributions to the oblique parameters $S$ and $T$. Similarly, the existence of the gauge-boson KK-resonances leads to a change in $G_{f}$, the four-Fermi coupling. We perform a $\chi^{2}$ test using the data on 22 such precision-measured observables, to find that the theory agrees very well with the low-energy data for $\lambda_{v} \lesssim 0.5$, and is virtually indistinguishable from the SM for $\lambda_{v} \lesssim 0.3$, both of which represent only a very small hierarchy between the Higgs vev and the UV cutoff.

It is instructive to consider the reason for and the circumstances of this agreement. For one, just as in the case of the graviton-tower, the coupling of the gauge boson-tower with the SM fermions are also somewhat suppressed. The consequent reduction in $\delta G_{f}$ obviously helps. However, much of $\Delta \chi^{2}$ accrues from the modification of the wave-function. It is here that this scenario is not very different from the five-dimensional analogue. Consequently, the limits on the KK-masses are very similar, the present scenario doing only marginally better. What is of more importance is that raising the KK-masses in the five-dimensional theory begins to call into doubt the semi-classical approximation that is the cornerstone of the treatment of the gravity sector. Indeed, even with the introduction of additional physics and a custodial symmetry, a fine tuning of $\mathcal{O}\left(10^{-2}\right)$ would be needed. In contrast, the sixdimensional theory studied here requires only a small fine tuning of $\mathcal{O}\left(\lambda_{v}\right)$. Furthermore, 
whereas the five-dimensional analogue would essentially push up all the resonances (except, maybe, the radion) above the reach of the LHC, this is not the case here. For, the Higgs resonances provide additional handles that can the model can be probed with. It would also be interesting to examine this sector at the LHC, but we postpone this to a later effort.

And, finally, we come to the issue of RG evolution. With the proliferation of states, naively, it would seem that the evolution would be much faster than is the case for the five-dimensional theory. This is not quite true, for a small $k$ means that the excitations in the $x_{5}$-directions are typically heavier. However, certain features (such as the loss of asymptotic freedom for the $\mathrm{SU}(2)$ interactions) are, understandably, quite similar to that in UED theories, but for the fact that the masses (and, hence, the thresholds) are non-uniformly spaced here. This, for example, leads to rapid changes in the sign of the beta-functions at certain intermediate points.

It might seem, overall, that by making the masses large, we have, essentially, decoupled the KK-sector. While this is forced upon us by the extremely good agreement of the low-energy observable with the SM expectations, it should be realized that the required masses, apart from being somewhat lower than is the case for the five-dimensional theory, are perfectly commensurate with the applicability of the semi-classical treatment of the gravitational sector, and does not need the introduction of additional symmetries (as the RS case does) to bring down the scale. However, if such a custodial symmetry is indeed imposed, the mass scale can be brought down and interesting signals may be seen at the LHC itself. We leave this for a future study. Also postponed is a thorough investigation of the Higgs sector, especially the consequences of our novel localization scheme wherein the scalar acquires a non-trivial classical configuration along a four-brane.

\section{Acknowledgments}

MTA would like to thank UGC-CSIR, India for assistance under Senior Research Fellowship Grant Sch/SRF/AA/139/F-123/2011-12. DC acknowledges partial support from the European Union FP7 ITN INVISIBLES (Marie Curie Actions, PITN-GA-2011-289442), and the Research and Development grant of the University of Delhi.

Open Access. This article is distributed under the terms of the Creative Commons Attribution License (CC-BY 4.0), which permits any use, distribution and reproduction in any medium, provided the original author(s) and source are credited.

\section{References}

[1] V.A. Rubakov and M.E. Shaposhnikov, Do We Live Inside a Domain Wall?, Phys. Lett. B 125 (1983) 136 [INSPIRE].

[2] M. Visser, An Exotic Class of Kaluza-Klein Models, Phys. Lett. B 159 (1985) 22 [hep-th/9910093] [INSPIRE].

[3] I. Antoniadis, A Possible new dimension at a few TeV, Phys. Lett. B 246 (1990) 377 [INSPIRE]. 
[4] M. Gogberashvili, Hierarchy problem in the shell universe model, Int. J. Mod. Phys. D 11 (2002) 1635 [hep-ph/9812296] [INSPIRE].

[5] M. Gogberashvili, Our world as an expanding shell, Europhys. Lett. 49 (2000) 396 [hep-ph/9812365] [INSPIRE].

[6] L. Randall and R. Sundrum, A Large mass hierarchy from a small extra dimension, Phys. Rev. Lett. 83 (1999) 3370 [hep-ph/9905221] [INSPIRE].

[7] L. Randall and R. Sundrum, An Alternative to compactification, Phys. Rev. Lett. 83 (1999) 4690 [hep-th/9906064] [INSPIRE].

[8] N. Arkani-Hamed, S. Dimopoulos and G.R. Dvali, The Hierarchy problem and new dimensions at a millimeter, Phys. Lett. B 429 (1998) 263 [hep-ph/9803315] [INSPIRE].

[9] I. Antoniadis, N. Arkani-Hamed, S. Dimopoulos and G.R. Dvali, New dimensions at a millimeter to a Fermi and superstrings at a TeV, Phys. Lett. B 436 (1998) 257 [hep-ph/9804398] [INSPIRE].

[10] A. Pomarol, Gauge bosons in a five-dimensional theory with localized gravity, Phys. Lett. B 486 (2000) 153 [hep-ph/9911294] [INSPIRE].

[11] H. Davoudiasl, J.L. Hewett and T.G. Rizzo, Bulk gauge fields in the Randall-Sundrum model, Phys. Lett. B 473 (2000) 43 [hep-ph/9911262] [INSPIRE].

[12] T.G. Rizzo and J.D. Wells, Electroweak precision measurements and collider probes of the standard model with large extra dimensions, Phys. Rev. D 61 (2000) 016007 [hep-ph/9906234] [INSPIRE].

[13] S. Chang, J. Hisano, H. Nakano, N. Okada and M. Yamaguchi, Bulk standard model in the Randall-Sundrum background, Phys. Rev. D 62 (2000) 084025 [hep-ph/9912498] [INSPIRE].

[14] C. Csáki, J. Erlich and J. Terning, The Effective Lagrangian in the Randall-Sundrum model and electroweak physics, Phys. Rev. D 66 (2002) 064021 [hep-ph/0203034] [INSPIRE].

[15] K. Agashe, A. Delgado, M.J. May and R. Sundrum, RS1, custodial isospin and precision tests, JHEP 08 (2003) 050 [hep-ph/0308036] [INSPIRE].

[16] B.A. Dobrescu and E. Poppitz, Number of fermion generations derived from anomaly cancellation, Phys. Rev. Lett. 87 (2001) 031801 [hep-ph/0102010] [INSPIRE].

[17] T. Appelquist, B.A. Dobrescu, E. Ponton and H.-U. Yee, Proton stability in six-dimensions, Phys. Rev. Lett. 87 (2001) 181802 [hep-ph/0107056] [INSPIRE].

[18] G. Burdman, B.A. Dobrescu and E. Ponton, Resonances from two universal extra dimensions, Phys. Rev. D 74 (2006) 075008 [hep-ph/0601186] [INSPIRE].

[19] B.A. Dobrescu, K. Kong and R. Mahbubani, Leptons and photons at the LHC: Cascades through spinless adjoints, JHEP 07 (2007) 006 [hep-ph/0703231] [INSPIRE].

[20] A. Freitas and K. Kong, Two universal extra dimensions and spinless photons at the ILC, JHEP 02 (2008) 068 [arXiv:0711.4124] [INSPIRE].

[21] D. Choudhury, A. Datta, D.K. Ghosh and K. Ghosh, Exploring two Universal Extra Dimensions at the CERN LHC, JHEP 04 (2012) 057 [arXiv:1109.1400] [INSPIRE].

[22] B.A. Dobrescu, D. Hooper, K. Kong and R. Mahbubani, Spinless photon dark matter from two universal extra dimensions, JCAP 10 (2007) 012 [arXiv:0706.3409] [INSPIRE]. 
[23] G. Cacciapaglia, A. Deandrea and J. Llodra-Perez, A Dark Matter candidate from Lorentz Invariance in 6D, JHEP 03 (2010) 083 [arXiv:0907.4993] [INSPIRE].

[24] G. Cacciapaglia, A. Deandrea and J. Llodra-Perez, The Universal Real Projective Plane: LHC phenomenology at one Loop, JHEP 10 (2011) 146 [arXiv:1104.3800] [InSPIRE].

[25] S. Randjbar-Daemi and M.E. Shaposhnikov, On some new warped brane world solutions in higher dimensions, Phys. Lett. B 491 (2000) 329 [hep-th/0008087] [INSPIRE].

[26] N. Kaloper, Origami world, JHEP 05 (2004) 061 [hep-th/0403208] [INSPIRE].

[27] T. Gherghetta and A. Kehagias, Anomaly free brane worlds in seven-dimensions, Phys. Rev. Lett. 90 (2003) 101601 [hep-th/0211019] [INSPIRE].

[28] Z. Chacko and A.E. Nelson, A Solution to the hierarchy problem with an infinitely large extra dimension and moduli stabilization, Phys. Rev. D 62 (2000) 085006 [hep-th/9912186] [INSPIRE].

[29] A.G. Cohen and D.B. Kaplan, Solving the hierarchy problem with noncompact extra dimensions, Phys. Lett. B 470 (1999) 52 [hep-th/9910132] [INSPIRE].

[30] R. Gregory, Nonsingular global string compactifications, Phys. Rev. Lett. 84 (2000) 2564 [hep-th/9911015] [INSPIRE].

[31] S.M. Carroll, S. Hellerman and M. Trodden, BPS domain wall junctions in infinitely large extra dimensions, Phys. Rev. D 62 (2000) 044049 [hep-th/9911083] [INSPIRE].

[32] N. Arkani-Hamed, L.J. Hall, D. Tucker-Smith and N. Weiner, Solving the hierarchy problem with exponentially large dimensions, Phys. Rev. D 62 (2000) 105002 [hep-ph/9912453] [INSPIRE].

[33] T. Gherghetta and M.E. Shaposhnikov, Localizing gravity on a stringlike defect in six dimensions, Phys. Rev. Lett. 85 (2000) 240 [hep-th/0004014] [INSPIRE].

[34] M. Giovannini, H. Meyer and M.E. Shaposhnikov, Warped compactification on Abelian vortex in six-dimensions, Nucl. Phys. B 619 (2001) 615 [hep-th/0104118] [INSPIRE].

[35] C.P. Burgess, J.M. Cline, N.R. Constable and H. Firouzjahi, Dynamical stability of six-dimensional warped brane worlds, JHEP 01 (2002) 014 [hep-th/0112047] [INSPIRE].

[36] M. Giovannini, Gauge field localization on Abelian vortices in six-dimensions, Phys. Rev. D 66 (2002) 044016 [hep-th/0205139] [INSPIRE].

[37] M. Giovannini, Scalar normal modes of higher dimensional gravitating kinks, Class. Quant. Grav. 20 (2003) 1063 [gr-qc/0207116] [INSPIRE].

[38] P. Kanti, R. Madden and K.A. Olive, 6-dimensional brane world model, Phys. Rev. D 64 (2001) 044021 [hep-th/0104177] [INSPIRE].

[39] T.G. Rizzo, Introduction to Extra Dimensions, AIP Conf. Proc. 1256 (2010) 27 [arXiv: 1003.1698] [INSPIRE].

[40] Z. Chacko, P.J. Fox, A.E. Nelson and N. Weiner, Large extra dimensions from a small extra dimension, JHEP 03 (2002) 001 [hep-ph/0106343] [INSPIRE].

[41] F. Chen, J.M. Cline and S. Kanno, Modified Friedmann Equation and Inflation in Warped Codimension-two Braneworld, Phys. Rev. D 77 (2008) 063531 [arXiv:0801.0226] [INSPIRE].

[42] K.L. McDonald, Little Randall-Sundrum Model and a Multiply Warped Spacetime, Phys. Rev. D 77 (2008) 124046 [arXiv:0804.0654] [INSPIRE]. 
[43] A.A. Saharian, Surface Casimir densities and induced cosmological constant in higher dimensional braneworlds, Phys. Rev. D 74 (2006) 124009 [hep-th/0608211] [INSPIRE].

[44] P. Midodashvili, Gravitational trapping of matters in $6 D$ and three fermion generations, Europhys. Lett. 66 (2004) 478 [INSPIRE].

[45] M. Gogberashvili and D. Singleton, Nonsingular increasing gravitational potential for the brane in 6-D, Phys. Lett. B 582 (2004) 95 [hep-th/0310048] [InSPIRE].

[46] M. Gogberashvili and D. Singleton, Brane in 6-D with increasing gravitational trapping potential, Phys. Rev. D 69 (2004) 026004 [hep-th/0305241] [INSPIRE].

[47] M. Gogberashvili, P. Midodashvili and D. Singleton, Fermion Generations from 'Apple-Shaped' Extra Dimensions, JHEP 08 (2007) 033 [arXiv:0706.0676] [INSPIRE].

[48] D. Choudhury and S. Sengupta, Living on the edge in a spacetime with multiple warping, Phys. Rev. D 76 (2007) 064030 [hep-th/0612246] [inSPIRE].

[49] ATLAS collaboration, Search for high-mass diphoton resonances in pp collisions at $\sqrt{s}=8 \mathrm{TeV}$ with the ATLAS detector, Phys. Rev. D 92 (2015) 032004 [arXiv:1504.05511] [INSPIRE].

[50] CMS collaboration, Search for High-Mass Diphoton Resonances in pp Collisions at $\sqrt{s}=8 \mathrm{TeV}$ with the CMS Detector, CMS-PAS-EXO-12-045 (2015) [INSPIRE].

[51] M.T. Arun, D. Choudhury, A. Das and S. SenGupta, Graviton modes in multiply warped geometry, Phys. Lett. B 746 (2015) 266 [arXiv:1410.5591] [INSPIRE].

[52] M.T. Arun and P. Saha, Gravitons in multiply warped scenarios - at $750 \mathrm{GeV}$ and beyond, Phys. Lett. B 746 (2015) 266 [arXiv: 1512.06335] [INSPIRE].

[53] ATLAS collaboration, Search for resonances decaying to photon pairs in $3.2 \mathrm{fb}^{-1}$ of $\mathrm{pp}$ collisions at $\sqrt{s}=13 \mathrm{TeV}$ with the ATLAS detector, ATLAS-CONF-2015-081 (2015) [INSPIRE].

[54] CMS collaboration, Search for new physics in high mass diphoton events in proton-proton collisions at $\sqrt{s}=13 \mathrm{TeV}$, CMS-PAS-EXO-15-004 (2015) [INSPIRE].

[55] M.T. Arun and D. Choudhury, Bulk gauge and matter fields in nested warping: I. The formalism, JHEP 09 (2015) 202 [arXiv: 1501.06118] [INSPIRE].

[56] M.E. Peskin and T. Takeuchi, Estimation of oblique electroweak corrections, Phys. Rev. D 46 (1992) 381 [INSPIRE].

[57] G. Altarelli and R. Barbieri, Vacuum polarization effects of new physics on electroweak processes, Phys. Lett. B 253 (1991) 161 [INSPIRE].

[58] Particle Data Group collaboration, K.A. Olive et al., Review of Particle Physics, Chin. Phys. C 38 (2014) 090001 [INSPIRE].

[59] M. Baak et al., The Electroweak Fit of the Standard Model after the Discovery of a New Boson at the LHC, Eur. Phys. J. C 72 (2012) 2205 [arXiv:1209.2716] [INSPIRE].

[60] M. Ciuchini, E. Franco, S. Mishima and L. Silvestrini, Electroweak Precision Observables, New Physics and the Nature of a 126 GeV Higgs Boson, JHEP 08 (2013) 106 [arXiv: 1306.4644] [INSPIRE].

[61] GFItTer Group collaboration, M. Baak et al., The global electroweak fit at NNLO and prospects for the LHC and ILC, Eur. Phys. J. C 74 (2014) 3046 [arXiv:1407.3792] [INSPIRE]. 
[62] SLD Electroweak Group, SLD Heavy Flavour Group, LEP Electroweak Working Group, OPAL, L3, DELPHI, ALEPH and SLD collaborations, S. Schael et al., Precision electroweak measurements on the $Z$ resonance, Phys. Rept. 427 (2006) 257 [hep-ex/0509008] [INSPIRE].

[63] K.R. Dienes, E. Dudas and T. Gherghetta, Grand unification at intermediate mass scales through extra dimensions, Nucl. Phys. B 537 (1999) 47 [hep-ph/9806292] [INSPIRE].

[64] G. Bhattacharyya, A. Datta, S.K. Majee and A. Raychaudhuri, Power law blitzkrieg in universal extra dimension scenarios, Nucl. Phys. B 760 (2007) 117 [hep-ph/0608208] [INSPIRE]. 